University of Nebraska - Lincoln

DigitalCommons@University of Nebraska - Lincoln

December 1990

\title{
Magnetism of rare-earth-transition-metal nanoscale multilayers. II. Theoretical analysis of magnetization and perpendicular magnetic anisotropy
}

\author{
Z.S. Shan \\ University of Nebraska - Lincoln \\ David J. Sellmyer \\ University of Nebraska-Lincoln, dsellmyer@unl.edu \\ Sitaram Jaswal \\ University of Nebraska, sjaswal1@unl.edu \\ Y.J. Wang \\ University of Nebraska - Lincoln \\ J.X. Shen \\ University of Nebraska - Lincoln
}

Follow this and additional works at: https://digitalcommons.unl.edu/physicssellmyer

Part of the Physics Commons

Shan, Z.S.; Sellmyer, David J.; Jaswal, Sitaram; Wang, Y.J.; and Shen, J.X., "Magnetism of rareearth-transition-metal nanoscale multilayers. II. Theoretical analysis of magnetization and perpendicular magnetic anisotropy" (1990). David Sellmyer Publications. 121.

https://digitalcommons.unl.edu/physicssellmyer/121

This Article is brought to you for free and open access by the Research Papers in Physics and Astronomy at DigitalCommons@University of Nebraska - Lincoln. It has been accepted for inclusion in David Sellmyer Publications by an authorized administrator of DigitalCommons@University of Nebraska - Lincoln. 


\title{
Magnetism of rare-earth - transition-metal nanoscale multilayers. II. Theoretical analysis of magnetization and perpendicular magnetic anisotropy
}

\author{
Z. S. Shan, ${ }^{*}$ D. J. Sellmyer, S. S. Jaswal, Y. J. Wang, ${ }^{\dagger}$ and J. X. Shen \\ Behlen Laboratory of Physics and Center for Materials Research and Analysis, University of Nebraska-Lincoln, \\ Lincoln, Nebraska 68588-0111
}

(Received 16 January 1990; revised manuscript received 30 April 1990)

\begin{abstract}
Systematic studies of rare-earth-transition-metal amorphous compositionally modulated films (CMF) permit the development of a detailed model for understanding the magnetization and perpendicular magnetic anisotropy and their distributions along the film normal. It is shown that the single-ion anisotropy of rare-earth ions with orbital angular momentum is the major contributor to the perpendicular anisotropy for this class of CMF. The calculated results agree well with the experimental data for $\mathrm{Dy} / \mathrm{Co}$ and $\mathrm{Tb} / \mathrm{Fe} \mathrm{CMF}$ when the individual layer thicknesses are small enough ( $<10 \AA$ ); these films have structures which can be characterized as compositionally modulated amorphous alloys.
\end{abstract}

\section{INTRODUCTION}

The magnetism and especially the perpendicular magnetic anisotropy (PMA) in compositionally modulated films (CMF) are of great interest because of both interface magnetic and low-dimensional effects, and also their promising properties with possible applications to perpendicular magnetic and magneto-optical recording. In recent years, a number of CMF have been studied. Some examples are $A^{\mathrm{RE}} / B^{\mathrm{TM}}\left(A^{\mathrm{RE}}=\mathrm{Tb}, \mathrm{Dy}, \mathrm{Nd}\right.$, and $\left.B^{\mathrm{TM}}=\mathrm{Co}, \mathrm{Fe}\right),{ }^{1-14} \mathrm{Pd} / \mathrm{Co},{ }^{15-22} \mathrm{Au} / \mathrm{Co},{ }^{23} \mathrm{Fe} / \mathrm{Ag},{ }^{24}$ and the results suggest that the CMF constitute a fertile area of pure and applied science, particularly in view of their controllable properties.

The existence of the compositional modulation of the constituent atoms offers an extra degree of freedom to control the local atomic environments in CMF structure, which permits the tailoring of magnetic properties, such as the perpendicular anisotropy, in artificially structured CMF. However, this also introduces complications in understanding the magnetic properties analytically because of the inhomogeneous distribution of constituents. Thus far, there has not been a thorough study of the micromagnetic structure of CMF: Sato ${ }^{1,2}$ suggested that the anisotropic distribution of $\mathrm{Tb}-\mathrm{Fe}$ pairs aligned perpendicular to the film plane was the most probable origin of the perpendicular anisotropy in amorphous $\mathrm{Tb} / \mathrm{Fe}$ multilayers, but his suggestion is only qualitative. Further, he does not indicate which mechanism is responsible for perpendicular anisotropy, e.g., the dipolarinteraction or single-ion anisotropy. Draaisma ${ }^{21}$ gave a phenomenological expression for the anisotropy energy based on the dipolar interaction for $\mathrm{Pd} / \mathrm{Co}$ multilayers, where the interfaces were assumed to be polycrystalline, however, Carcia ${ }^{15}$ and de Broeder ${ }^{18}$ proposed that the stress-induced anisotropy contributes to PMA for this system. Suzuki ${ }^{25}$ and Yoshihara et al. ${ }^{26-29}$ claimed that single-ion anisotropy is the main source of anisotropy, but their discussion was confined to homogeneous alloys.
Suzuki et $a l^{7}$ suggested that for Nd/Co CMF the perpendicular anisotropy is induced from $\mathrm{Nd}$ atoms which are adjacent to Co atoms in the vicinity of the interface. It seems that two points are oversimplified in their discussion. (1) The modulated composition was replaced by a fixed composition of $\mathrm{Nd}-\mathrm{Co}$ alloy during the mean-field fitting, i.e., the compositionally modulated structure has not been considered there. (2) The mechanism responsible for anisotropy has not been pointed out clearly. To our knowledge there have been no attempts to describe the micromagnetic structure of an $A^{\mathrm{RE}} / B^{\mathrm{TM}}$ amorphous CMF in detail, namely, the distribution of magnetization and anisotropy, the origin of the anisotropy, and the relationship between anisotropy and the distributions of magnetization and constituent atoms.

We have performed systematic studies of $A^{\mathrm{RE}} / B^{\mathrm{TM}}$ CMF including the case of $A^{\mathrm{RE}}=\mathrm{Tb}, \mathrm{Dy}, \mathrm{Gd}, \mathrm{Er}, \mathrm{Nd}$, and $B^{\mathrm{TM}}=\mathrm{Fe}, \mathrm{Co}, \mathrm{Ni}^{8-14,30}$ These experiments have permitted us to develop a model ${ }^{13}$ in terms of which the detailed properties, including PMA, can be understood. A modified mean-field model is used to analyze magnetization, and a single-ion model is used for analyzing the anisotropy. The calculated data based on these models agree with the experimental results reasonably well, and the origin of the anisotropy will be clarified.

\section{MAGNETIZATION}

The mean-field model has been used extensively to calculate the magnetization of $A^{\mathrm{RE}}-B^{\mathrm{TM}}$ homogeneous alloys. $^{31-35}$ This method is adopted to the CMF structure in the present paper. The main physical ideas are as follows. (1) The compositional dependence of magnetization for $A_{1-c}^{\mathrm{RE}} / B_{c}^{\mathrm{TM}}$ homogeneous alloys is calculated by mean-field theory and fitted to the experimental data. (2) The $A^{\mathrm{RE}} / B^{\mathrm{TM}} \mathrm{CMF}$ is divided into thin slices each of which can be regarded as a two-dimensional amorphous film as shown in Fig. 1(a). (3) A trial distribution of TM concentration, e.g., a sinusoidal function, is assumed for 


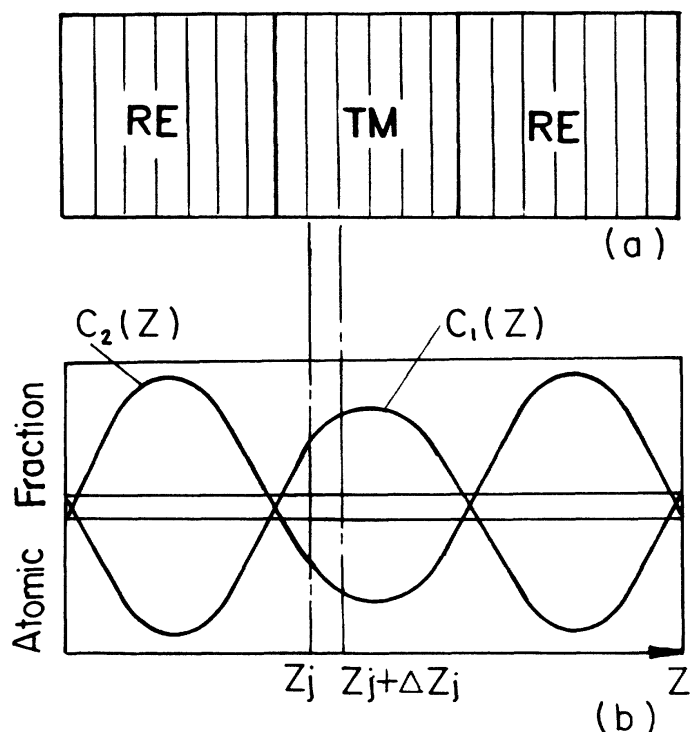

FIG. 1. The schematic diagram of $A^{\mathrm{RE}} / B^{\mathrm{TM}} \mathrm{CMF}$ regarded as the synthesis of homogeneous $A_{1-c}^{\mathrm{RE}} B_{c}^{\mathrm{TM}}$ slices (a), and the concentration distributions of Dy and Co along the film normal.

the thin layer-thickness CMF as shown in Fig. 1(b) because of the interdiffusion between RE and TM atoms, and also that the results of small angle $\mathrm{x}$-ray diffraction pattern shows only the first-order peak for Dy/Co, $\mathrm{Dy} / \mathrm{Fe}, \mathrm{Tb} / \mathrm{Fe} \mathrm{CMF}$ with thin individual layer thicknesses (see Fig. 2 in paper I and Refs. 36 and 30). The magnetization $\sigma_{j}$ of the $j$ th slice is determined and then the resultant magnetization is obtained by taking the average of $\sigma$, over the whole volume of the CMF. (4) The trial distribution is adjusted by repeatedly comparing the calculated results with the experimental data to achieve a good fit. This fitting gives the possible distributions of the constituent atomic fraction, the total magnetization, and its constituent magnetization along the film normal. In the following sections the calculation procedures and discussions to obtain a deeper physical understanding of the properties are described.

\section{A. Magnetization for homogeneous $A^{\mathrm{RE}}-B^{\mathrm{TM}}$ alloys}

For homogeneous $A_{1-c}^{\mathrm{RE}} B_{c}^{\mathrm{TM}}$ alloys, the spontaneous magnetization $\sigma_{s}$ is governed by the following set of equations based on the mean-field model. ${ }^{31}$

\section{Basic equations for magnetization of $A_{1-c}^{R E} B_{c}^{T M}$ alloys}

In the mean-field model we have

$$
\begin{aligned}
& \sigma_{s}=N \mu_{B}\left(C_{1} g_{1} \bar{S}_{1} \pm C_{2} g_{2} \bar{S}_{2}\right), \\
& \bar{S}_{1}=S_{1} B_{S_{1}}\left(\frac{g_{1} \mu_{B} S_{1} H_{1}}{k_{B} T}\right), \\
& \bar{S}_{2}=S_{2} B_{S_{2}}\left(\frac{g_{2} \mu_{B} S_{2} H_{2}}{k_{B} T}\right),
\end{aligned}
$$

$$
\begin{aligned}
& H_{1}=2\left(\frac{J_{11} Z_{11} \bar{S}_{1}}{g_{1} \mu_{B}}+\frac{J_{12} Z_{12} \bar{S}_{2}}{g_{1} \mu_{B}}\right), \\
& H_{2}=2\left(\frac{J_{21} Z_{21} \bar{S}_{1}}{g_{2} \mu_{B}}+\frac{J_{22} Z_{22} \bar{S}_{2}}{g_{2} \mu_{B}}\right),
\end{aligned}
$$

where the subscript $i(i=1,2)$ refers to TM and RE subnetworks and $+(-)$ sign refers to light RE (heavy RE), and $C_{i}$ is the atomic fraction of the $i$ th constituent, so that $C_{2}=1-C_{1}$ (we will use $C$ simply to denote $C_{1}$ below), $g_{i}$ is the gyromagnetic factor of the $i$ th atom and $H_{i}$ is the effective field of the $i$ th subnetwork. $\bar{S}_{i}$ and $S_{i}$ are the effective subnetwork spin at room and zero temperatures, respectively. $J_{i j}$ are the exchange constants and $Z_{i j}$ is the number of nearest neighbors. $N$ is the total number of atoms per unit volume, $k_{B}$ is the Boltzmann constant, and $\mu_{B}$ is the Bohr magneton. $B_{S_{1}}$ denotes the Brillouin function.

We take Dy/Co CMF as an example, but this method is also suitable for the other similar $A^{\mathrm{RE}} / B^{\mathrm{TM}}$ systems.

In the fitting procedure we first calculate the Co compositional dependence of magnetization for amorphous $\mathrm{Dy}_{1-c} \mathrm{Co}_{c}$ in terms of Eqs. (1)-(5). The calculated $\sigma_{s}$ can be fitted optimally to the experimental $\sigma_{s}$ over a wide range of composition $C$ with the following parameters: $J_{11}=(2.35 C-0.95) \times 10^{-14} \mathrm{erg}, J_{22}=8.00 \times 10^{-17} \mathrm{erg}$, $J_{12}=-(1.3-0.6 C) \times 10^{-15} \quad \mathrm{erg}, \quad g_{1}=2, \quad S_{1}=0.735$, $g_{2}=\frac{4}{3}, S_{2}=5.47$. These values are subject to a certain degree of nonuniqueness. For example, the value of $g_{2} S_{2}=7.3$ which is smaller than the value appropriate for free $\mathrm{Dy}^{3+}$ ion $\left(g_{2} S_{2}=10\right)$. This is interpreted as decreasing from a fanning of the Dy moments into a cone of half angle 63 degrees. The origin of this is the wellknown random anisotropy effect. In addition, one could imagine allowing the Co spin, the $J_{i j}$ values, and the coordination numbers to be functions of composition. However, our assumptions, which correspond to those used by other workers who have treated homogeneous $A^{\mathrm{RE}}-B^{\mathrm{TM}}$ alloys, are the simplest ones which lead to

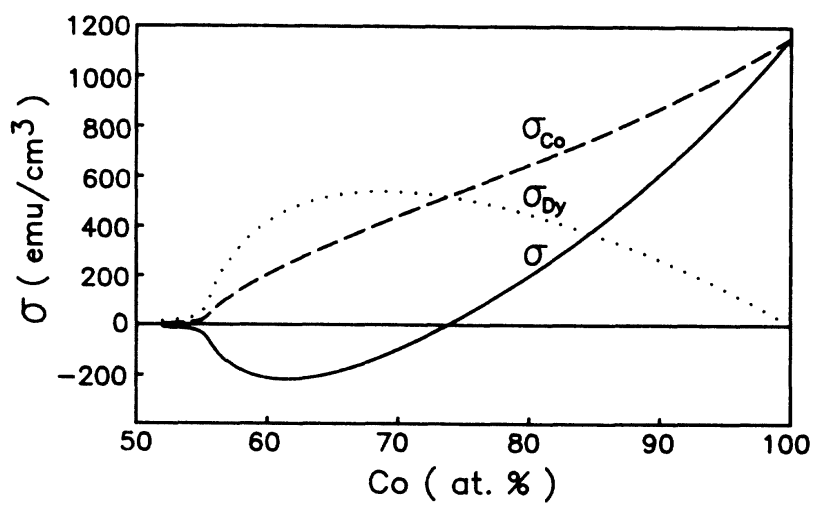

FIG. 2. The Co concentration dependence of magnetization for Dy-Co alloys: the total magnetization $\sigma_{s}$, Dy-subnetwork magnetization $\sigma_{\mathrm{Dy}}$, and Co-subnetwork magnetization $\sigma_{\mathrm{Co}}$. 
physically reasonable results.

Using these data, the magnetization curves $\sigma_{s}(C)$, $\sigma_{1}(C)$, and $\sigma_{2}(C)$, i.e., the total spontaneous magnetization, the magnetization of Co and Dy constituents, are shown in Fig. 2. Here

$$
\sigma_{1}(C)=N C \mu_{B} g_{1} \bar{S}_{1}, \quad \sigma_{2}(C)=N(1-C) \mu_{B} g_{2} \bar{S}_{2} .
$$

These curves are the basis of describing the magnetic structure of Dy/Co CMF.

\section{Discussion}

a. Physical origin of the magnetic ordering for Dy-Co binary alloys. Physically the Dy subnetwork is disordered magnetically at room temperature if the Co subnetwork does not exist. It is the exchange interaction between Co and Dy which causes magnetic ordering for Dy subnetwork. If there is no such exchange interaction, i.e., $J_{12}=J_{21}=0$, obviously Eqs. (2) and (3) become linearly independent and $\bar{S}_{2}$ becomes zero because the Dy moment is disordered at room temperature.

Further analysis shows that the effective subnetwork spin $\bar{S}_{i}$ depends on the argument $y_{i}=g_{i} \mu_{B} S_{i} H_{i} / k_{B} T$ [see Eqs. (2) and (3)]. If $y_{i}$ is less than the critical value corresponding to the Curie temperature, the $i$ th subnetwork will be disordered magnetically. For the binary Dy-Co alloys, two parameters affect the argument $y$ at least: they are temperature $T$, and effective field $H_{i}$ [see Eqs. (4) and (5)] which in turn depend on $\bar{S}_{i}, J_{i j}$, and the composition $C$ through the expression of $Z_{i j}$ as ${ }^{31}$

$$
Z_{11}=Z_{21}=12 C, \quad Z_{22}=Z_{12}=12(1-C),
$$

where $C$ is the atomic fraction of Co atoms. In the above equations the approximation of 12 -fold coordination has been adopted.

Using these relations, Eqs. (4) and (5) may be written as

$$
\begin{aligned}
& H_{1}=\frac{24}{g_{1} \mu_{B}}\left[J_{11} C \bar{S}_{1}-\left|J_{12}\right|(1-C) \bar{S}_{2}\right], \\
& H_{2}=\frac{24}{g_{2} \mu_{B}}\left[\left|J_{12}\right| C \bar{S}_{1}+J_{22}(1-C) \bar{S}_{2}\right],
\end{aligned}
$$

and we notice that $J_{11}>0, J_{22}>0$, and $J_{12}=J_{21}<0$ for heavy $A^{\mathrm{RE}} / B^{\mathrm{TM}}$.

The compositional dependences of $H_{i}$ and $\bar{S}_{1}$, and consequently the argument $y$ are of great importance in the interpretation of the origin and main features of magnetization curves in Fig. 2.

b. The compositional dependence of the subnetwork internal field. the compositional dependence of the calculated internal field of Dy and Co subnetworks, i.e., the $H_{\mathrm{Dy}}(C)$ and $H_{\mathrm{Co}}(C)$, is illustrated in Fig. 3. It is seen that (1) the internal field $H_{\mathrm{Co}}(C)$ increases linearly with composition $C$ over a wide range and its maximum value is about $1.3 \times 10^{7}$ Oe. (2) The internal field $H_{\mathrm{Dy}}(C)$ is much smaller than that of $H_{\mathrm{Co}}(C)$ and its value is about $10^{6}$ Oe over a wide range of $C$. (3) To make a numerical estimation it is reasonable to ignore the external applied field $H_{a}$ for large values of $C$, e.g., $C>65$ at. \%. In the case of small $C$, either the external field $H_{a}$ has to be in-

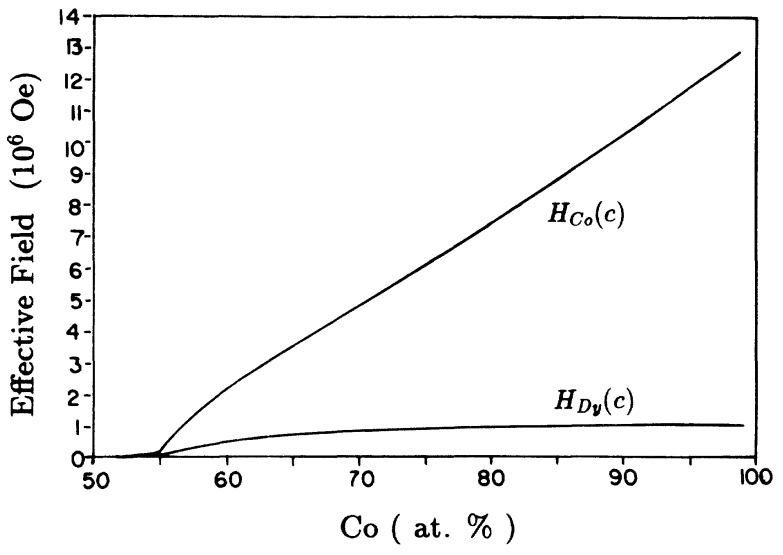

FIG. 3. The Co concentration dependence of subnetwork internal field $H_{\mathrm{Co}}$ and $H_{\mathrm{Dy}}$ at $300 \mathrm{~K}$.

volved or the spontaneous magnetization $\sigma_{s}$ should be adopted.

c. The compositional dependence of the effective subnetwork spins. The compositional dependence of the calculated subnetwork spins of $\bar{S}_{\mathrm{Dy}}(C)$ and $\bar{S}_{\mathrm{Co}}(C)$ is illustrated in Fig. 4. Both of them change their values smoothly for $C>75$ at. \%, and decrease rapidly as $C$ becomes less than $\sim 65$ at. $\%$. Finally they are disordered at $C \sim 53$ at. $\%$. We notice that both $\bar{S}_{\mathrm{Dy}}$ and $\bar{S}_{\mathrm{Co}}$ change smoothly through the compensation point at $C \simeq 74$ at. $\%$. This feature implies that both Dy and Co subnetworks are ordered magnetically at the compensation point, but their magnetic moments cancel each other because of antiferromagnetic coupling.

Figures 2-4 illustrate the pictorial behavior of the compositional dependence of magnetization, internal field, and spin, and this is valuable to understand the micromagnetic characteristics of the Dy-Co alloys.

The general behavior of $\sigma_{\mathrm{Co}}$ and $\sigma_{\text {Dy }}$ can be interpreted in terms of Eqs. (7) and (8) and Figs. 3 and 4. The internal field $H_{1}$ increases monotonically as $C$ increases (see Fig. 3) and consequently $\sigma_{\text {Co }}$ increases correspondingly. This character also can be seen in Eq. (7) because

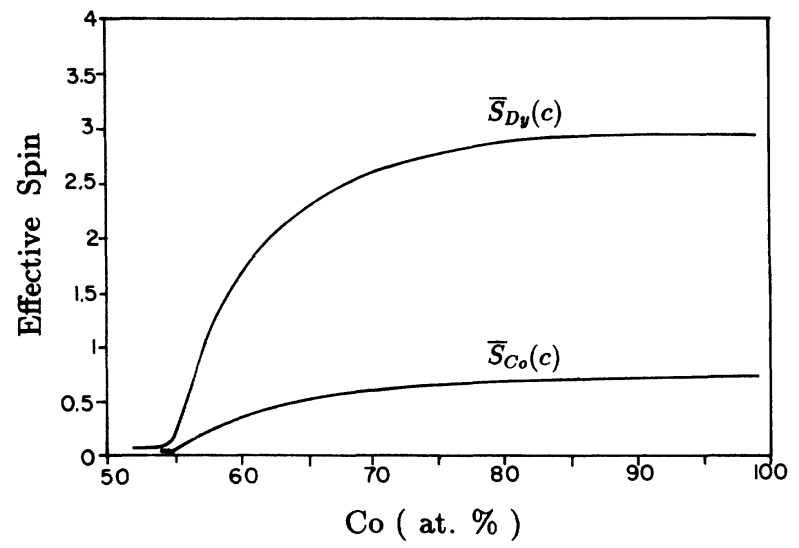

FIG. 4. The Co concentration dependence of the subnetwork spins $\bar{S}_{\mathrm{Dy}}$ and $\bar{S}_{\mathrm{Co}}$ at $300 \mathrm{~K}$. 
the term $J_{11} C \bar{S}_{1}$ dominates in comparison with the term $\left|J_{12}\right|(1-C) \bar{S}_{2}$ and becomes larger as $C$ increases. At small $C$, which is about 53 at. $\%$ in Fig. 2, the Co subnetwork is disordered because $J_{12}(1-C) \bar{S}_{2}$ term gives an appreciable negative contribution so that $H_{1}$ becomes less than the critical value corresponding to the Curie temperature. The behavior of the compositional dependence $\sigma_{\text {Dy }}$ (see Fig. 2) follows because $\mathrm{H}_{2}$ is small at small $C$ and at large $C$ the factor $(1-C)$ in Eq. (6) reduces the $\sigma_{\mathrm{Dy}}$ value. Consequently $\sigma_{\mathrm{Dy}}$ has larger values at intermediate $C$ and vanishes at both small and large values, as shown in Fig. 2. This may also be seen in Eq. (8) wherein $\mathrm{H}_{2}$ is small at small $\mathrm{C}$ because the term $\left|J_{12}\right| C \bar{S}_{1}$ gives a small contribution. At large $C$, each Dy atom has large spin $\bar{S}_{2}$ (see Fig. 4). The decrease of $\sigma_{\text {Dy }}$ value is because of the small Dy atomic fraction $(1-C)$ [see Eq. (6)].

\section{B. Magnetization and its distribution for $A^{\mathrm{RE}} / B^{\mathrm{TM}} \mathrm{CMF}$}

To determine the magnetization of the $A^{\mathrm{RE}} / B^{\mathrm{TM}}$ CMF we evaluate the magnetizations $\sigma_{s j}, \sigma_{1 j}$, and $\sigma_{2 j}$ of the $j$ th slice from Fig. 2 (assume that $A^{\mathrm{RE}}=\mathrm{Dy}$, $B^{\mathrm{TM}}=\mathrm{Co}$ ) if the trial distributions of Co and Dy concentrations (at. number $/ \mathrm{cm}^{3}$ ) along the film normal direction [see Fig. 1(b)] are assumed. Then the $C$ value of each slice can be calculated and the magnetization of the CMF is equal to

$$
\sigma_{s}=\frac{1}{\lambda} \sum_{j} \sigma_{s j} \Delta Z_{J}=\frac{1}{\lambda} \sum_{j}\left(\sigma_{1 j}-\sigma_{2 j}\right) \Delta Z_{j}
$$

where $\lambda$ is the bilayer thickness and $\Delta Z_{j}$ the thickness of the $j$ th slice. The parameters of the distribution function were adjusted until the $\sigma_{s}$ values calculated from Eq. (9) fitted the experimental data within the given error (1 $\mathrm{emu} / \mathrm{cm}^{3}$ in this work).

For $\mathrm{Dy} / \mathrm{Co}, \mathrm{Dy} / \mathrm{Fe}$, and $\mathrm{Tb} / \mathrm{Fe} \mathrm{CMF}$ of the thin individual layer thickness, the distribution of the constituent atoms has the sinusoidal form

$$
\eta_{J}(Z)=A_{j 0} \pm A_{j} \sin \left(\frac{2 \pi Z}{\lambda}\right),
$$

where $j=1$ and "+" sign refer to $\mathrm{TM}$, and $j=2$, and "- " sign refer to RE and the $Z$ axis is along the film normal. $\boldsymbol{A}_{j 0}$ is a constant and $\boldsymbol{A}_{j}$ the modulation amplitude of the $j$ th constitute concentration.

When the parameters $A_{j 0}$ and $A_{j}$ are determined by the optimum fitting, the distributions of the constituent atomic fraction, the total magnetization $\sigma_{s}(Z)$, and the constituent magnetization $\sigma_{1}(Z)$ and $\sigma_{2}(Z)$ along the film normal are obtained.

To ensure that the fitting is reasonable, some constraints should be considered. (1) The conservation of the total number of TM and RE atoms is necessary during the process of assuming the trial distributions of TM and RE concentrations. (2) The summation of TM and RE atomic fractions is equal to unity. (3) The trial distribution curves of TM and RE have to be transferred smoothly through the nominal boundary. These constraints have to be obeyed to choose the trial distribution of TM and RE concentration. Further details of the constraints imposed are described in Appendix A.

Detailed examples of the magnetization distributions for $\mathrm{Dy} / \mathrm{Co}$ and $\mathrm{Tb} / \mathrm{Fe} \mathrm{CMF}$ are discussed in Sec. IV.

\section{ANISOTROPY}

\section{A. The origin of the anisotropy in amorphous $A^{\mathrm{RE}} / B^{\mathrm{TM}} \mathrm{CMF}$}

Many efforts have been made to investigate magnetic anisotropy and its origin in thin films. In principle, the necessary conditions to create PMA include the following.

(1) The anisotropic distributions of the constituent atoms which are determined by (a) the crystal structure for the crystalline RE (or TM) layers or the artificial multilayered structure which follows from the fabrication procedure, (b) the strain induced by the mismatch between the substrate and the $A^{\mathrm{RE}} / B^{\mathrm{TM}} \mathrm{CMF}$, and the tension (or compression) exerted on one layer (say RE layer) by its neighboring layers (say TM layers), and (c) the CSRO (compositional short-range order) and bondorientational order. ${ }^{37,38}$

(2) The interactions among the constituent atoms which include mainly the following two interactions: (a) magnetic dipolar interactions and (b) spin-orbit interactions which lead to single-ion anisotropy.

As outlined above, it is clear that once the anisotropic atomic structure is determined, this will then affect the magnitudes of both of the possible origins of PMA. Often, three sources of anisotropy are mentioned as candidates for PMA of thin films. These include dipolarinteraction,,$^{39,40}$ single-ion anisotropy,,$^{25-29,41,42}$ and stress-induced or "magnetostrictive" anisotropy. ${ }^{15,18}$ In fact, the third of these involves PMA induced by the distortion of the structure resulting from strains of the magnetic layers by the nonmagnetic layers or by the substrate.

The following three facts suggest that the main magnetic origin of PMA is due to single-ion anisotropy, as opposed to dipolar interactions, for amorphous $A^{\mathrm{RE}} /$ $B^{\mathrm{TM}}\left(A^{\mathrm{RE}}=\mathrm{Dy}, \mathrm{Tb}\right.$, and $\left.B^{\mathrm{TM}}=\mathrm{Fe}, \mathrm{Co}\right) \mathrm{CMF}$.

(1) Figure 5 illustrates our experimental results for the behavior of $K_{u}$ for $A^{\mathrm{RE}} / B^{\mathrm{TM}} \mathrm{CMF}$ ( $A^{\mathrm{RE}}=\mathrm{Tb}$, Dy, Gd, and $B^{\mathrm{TM}}=\mathrm{Fe}, \mathrm{Co}$ ). This figure demonstrates that the anisotropy $K_{u}$ for $\mathrm{Dy} / \mathrm{Fe}, \mathrm{Dy} / \mathrm{Co}$, and $\mathrm{Tb} / \mathrm{Fe} \mathrm{CMF}$ is roughly an order of magnitude larger than that of $\mathrm{Gd} / \mathrm{Fe}$ CMF. The $K_{u}$ of Gd/Co CMF is even smaller than that of $\mathrm{Gd} / \mathrm{Fe} \mathrm{CMF}$. Since, to first order, the $\mathrm{Gd}$ atom has no single-ion anisotropy, it is reasonable to attribute the main origin of the perpendicular anisotropy of the Dy and $\mathrm{Tb}$ CMF to single-ion anisotropy of $\mathrm{Dy}$ and $\mathrm{Tb}$ atoms.

(2) The dipolar-interaction model has been successful in explaining the perpendicular anisotropy in terms of an anisotropy parameter " $p$ " for Gd-Co amorphous alloys. ${ }^{39,40}$ Here $p$ is a measure of the anisotropy in the distribution of unlike nearest-neighbor pairs, and its value is only about 0.015 for bias-sputtered Gd-Co. We used this model, which has been modified, for Dy/Co CMF, and found that the average value $\langle p\rangle$ is about 0.2 , and its peak value may reach 0.4 , because the $K_{u}$ value of 


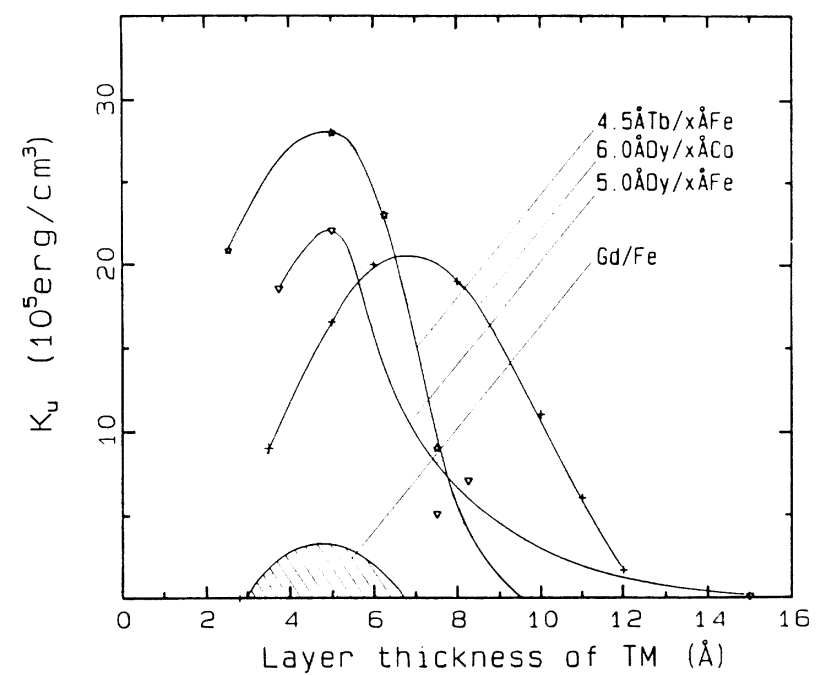

FIG. 5. The anisotropy for $(4.5 \AA \mathrm{Tb}) /(X \AA \mathrm{Fe}),(5 \AA \mathrm{Dy}) /(X$ $\AA \mathrm{Fe}),(6 \AA \mathrm{Dy}) /(X \AA \mathrm{Co})$, and $\mathrm{Gd} / \mathrm{Fe} \mathrm{CMF}$. The anisotropy data of $(2.3 \AA \mathrm{Gd}) /(3 \AA \mathrm{Fe}),(3 \AA \mathrm{Gd}) /(3 \AA \mathrm{Fe}),(3 \AA \mathrm{Gd}) /(5 \AA$ $\mathrm{Fe})$, etc., are in the shaded area.

Dy/Co CMF is an order magnitude larger than that of Gd/Co alloy. We noticed the $p$ value seems unreasonably large, which implies that the dipolar-interaction model is inadequate for $A^{\mathrm{RE}} / B^{\mathrm{TM}} \mathrm{CMF}\left(A^{\mathrm{RE}}=\mathrm{Dy}, \mathrm{Tb}\right.$, $\left.B^{\mathrm{TM}}=\mathrm{Co}, \mathrm{Fe}\right)$. But the dipolar-interaction model seems to be appropriate for $\mathrm{Gd} / B^{\mathrm{TM}} \mathrm{CMF}\left(B^{\mathrm{TM}}=\mathrm{Fe}, \mathrm{Co}\right.$ ).

(3) We have discovered that detailed layer-thickness dependence of the perpendicular anisotropy, as exhibited in Fig. 5, can be understood on the basis of the following expression:

$$
K_{u}=\xi \frac{A}{\lambda}\left\langle\sigma_{z}^{2}\right\rangle f\left(\frac{l_{1}}{l_{2}}\right),
$$

where $\left\langle\sigma_{z}^{2}\right\rangle$ is the average of magnetization squared of the rare-earth subnetwork, $\xi$ a constant, " $A$ " the peak to peak compositional modulation of the TM, $\lambda$ the bilayer thickness, and $f\left(l_{1} / l_{2}\right)$ is a smooth function depending on the ratio of TM layer thickness to RE layer thickness. $f\left(l_{1} / l_{2}\right)=1$ when the TM and RE have equal layer thickness. Equation (11) can be derived in terms of a single-ion model and this is a strong evidence that singleion anisotropy of the rare-earth atom is the main source of the perpendicular anisotropy.

The subject of PMA and its origin via crystal-field interaction in $A^{\mathrm{RE}} / B^{\mathrm{TM}}$ multilayers has been considered recently by Baczewski et al. ${ }^{42}$ and Fert. ${ }^{43}$ In addition, Lee et al. ${ }^{44}$ and den Broeder et al. ${ }^{45}$ have discussed PMA in $\mathrm{Co} / \mathrm{Au}$ and $\mathrm{Ni} / \mathrm{Pd}$ multilayers on the basis of magnetostrictive effects.

In the next section, Eq. (11) is derived briefly based on single-ion model assuming TM and RE have equal layer thickness. The derivation for the general case is given in Appendix B.

\section{B. The anisotropy for $A^{\mathrm{RE}} / B^{\mathrm{TM}} \mathrm{CMF}$}

Equation (11) can be obtained as follows. For the $i$ th slice of the CMF, the local single-ion anisotropy is given by (see Appendix B)

$$
K(i) \propto \alpha_{J}\left\langle r^{2}\right\rangle O_{2}^{0} A_{2}^{0},
$$

where $\alpha_{J}$ is Steven's factor, $\left\langle r^{2}\right\rangle$ the average radius squared of the $4 f$ orbit, $O_{2}^{0}$ is Steven's operator $O_{n}^{m}$ with $n=2$ and $m=0$. $O_{2}^{0}$ is a factor proportional to $J_{z}^{2}$ if $Z$ is the easy-axis direction. $A_{2}^{0}$ is a crystal-field term which is given by

$$
A_{2}^{0} \propto \sum_{j} \frac{q_{j}\left(3 \cos ^{2} \theta_{j}-1\right)}{r_{j}^{3}} .
$$

Then Eq. (10) becomes

$$
K(i) \propto \alpha_{J}\left\langle\sigma_{z}^{2}\right\rangle_{i} \sum_{j} \frac{q_{j}\left(3 \cos ^{2} \theta_{J}-1\right)}{r_{j}^{3}},
$$

where $j$ is the index of the $j$ th ion in the neighborhood of the RE ion at the $i$ th slice. The summation is over the neighboring ions with charge $q_{j}$ and distance $r_{j}$ from RE ion and $\theta_{j}$ is the angle with respect to the moment direction [see Fig. 6(a)]. The average notation \langle\rangle$_{i}$ means the statistical average of the RE ion over the $i$ th slice. In the above derivation the relation " $\sigma_{z} \propto J_{z}$ " has been used.

For the amorphous CMF structure the sum over $j$ in Eq. (13) has to be replaced by an integral weighted with an anisotropic probability function. ${ }^{25}$ This function for

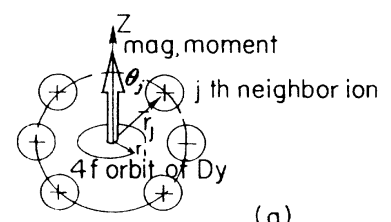

(a)

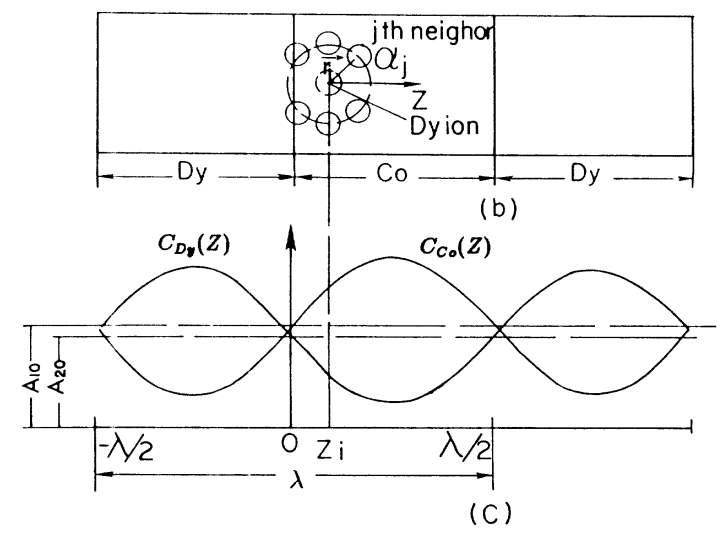

FIG. 6. The crystal field created by the neighbor ions (a), the local anisotropy $K(i)$ at $Z_{l}\left(\right.$ b). The resultant anisotropy $K_{u}$ is the average of $K(i)$ over the whole volume of CMF. The concentration distribution $C_{j}(j=1$ refers to $\mathrm{Co}$, and $j=2$ refers to Dy) along the film normal (c). 
CMF structure may be expressed as

$$
P_{i j}(\mathbf{r})=\eta_{,} R_{j}(r)\left[1+\beta_{j}(r) \cos \alpha_{j}+\cdots\right],
$$

where $\eta_{j}$ is the atomic fraction of the $j$ th surrounding ion consisting of RE and TM ions which have the sinusoidal form as shown in Eq. (10). $R_{J}(r)$ gives the isotropic part of the distribution, while $\beta_{j}(r)$ is the lowest-order anisotropic contribution. The parameter $\beta_{j}$, the anisotropy in the pair distribution function, is determined by the structural inhomogeneities produced by stress at the interfaces and chemical short-range order.

In the CMF structure $C_{j}$ varies along the film normal, and then

$P_{l J}(\mathbf{r}) \simeq\left[\eta_{J}\left(Z_{i}\right)+\eta_{j}^{\prime}\left(Z_{l}\right) r \cos \alpha_{j}\right] R_{j}(r)\left[1+\beta_{j}(r) \cos \alpha_{J}\right]$,

where the relation $\Delta Z_{l}=r \cos \alpha$, is adopted, i.e., the RE ion at the $i$ th slice is assumed to be the origin of the spherical coordinate and the polar axis is along the $Z$ direction, i.e., the film normal direction. Then the local anisotropy $K(i)$ of a $\mathrm{RE}$ ion at the $i$ th slice is found as follows:

$$
\begin{aligned}
K(i) & \propto \alpha_{J}\left\langle r^{2}\right\rangle\left\langle\sigma_{z}^{2}\right\rangle_{l} \int \frac{\left(3 \cos ^{2} \theta-1\right) \rho_{J}}{r_{J}^{3}} P_{l \jmath}(r) d \Omega_{J} \\
& \propto K_{0}\left\langle\sigma_{z}^{2}\right\rangle_{l}\left(\frac{A_{j}}{\lambda}\right) \cos \left(\frac{2 \pi Z_{l}}{\lambda}\right),
\end{aligned}
$$

where

$$
K_{0}=\alpha_{J}\left\langle r^{2}\right\rangle\left(\frac{\rho_{1} R\left(r_{1}\right) \beta_{1}}{r_{1}^{2}}-\frac{\rho_{2} R\left(r_{2}\right) \beta_{2}}{r_{2}^{2}}\right) .
$$

In the above derivation, the integral is calculated over the neighboring atoms with charge density $\rho_{j}$ and distance $r_{j}$ from RE ion, usually nearest-neighbor atoms only, and $\rho_{j}, R\left(r_{j}\right)$, and $\beta_{j}$ are assumed to be different, but constant for the $\operatorname{TM}(j=1)$ and $\operatorname{RE}(j=2)$ ions.

The anisotropy $K_{u}$ of the $A^{\mathrm{RE}} / B^{\mathrm{TM}} \mathrm{CMF}$ can be obtained as the average of $K(i)$ over all the slices as shown in Fig. 6(b). If the magnetization $\sigma_{z}$ changes smoothly along the film normal, which is true for the sample with thin layer thickness, $K_{u}$ can be written as

$$
K_{u}=\xi\left\langle\sigma_{z}^{2}\right\rangle\left(\frac{A}{\lambda}\right)
$$

as has been indicated in Eq. (11) while $f\left(l_{1} / l_{2}\right)=1$. A detailed derivation from Eq. (10) to Eq. (17) is given in Appendix B. The data for $\left\langle\sigma_{z}^{2}\right\rangle$ and " $A$ " can be obtained from Sec. II.

An important feature following from Eq. (16) is that the local anisotropy $K(i)$ is proportional to $\cos \left(2 \pi Z_{l} / \lambda\right)$; since the cosine function has its peak value at $Z_{l}=0$, i.e., the nominal boundary position, the interface region will give the main contribution to the anisotropy.

\section{ANALYSES FOR Dy/Co AND Tb/Fe CMF}

Physically all the experimental data are the results of statistical average over the whole sample. However, it is desirable to clarify the origin of the perpendicular anisotropy and obtain the distributions of magnetization and anisotropy since the composition of samples is modulated along the film normal. Two examples of analyses for $(6 \AA$ $\mathrm{Dy}) /(X \AA \mathrm{Co})$ and $(4.5 \AA \mathrm{Tb}) /(X \AA \mathrm{Fe})$ are given below. It will be seen that the calculated results agree well with the experimental data and some micromagnetic information can be obtained based on this model.

\section{A. Analysis for Dy/Co CMF}

The distributions of the magnetizations (the total magnetization $\sigma$, Co- and Dy-subnetwork magnetization $\sigma_{\text {Co }}$ and $\left.\sigma_{\mathrm{Dy}}\right)$ for $(6 \AA \mathrm{Dy}) /(3.5 \AA \mathrm{Co})$ and $(6 \AA \mathrm{Dy}) /(6 \AA \mathrm{Co})$ are shown in Fig. 7. It is seen that $\sigma_{\text {Dy }}$ dominates in Dy region and $\sigma_{\text {Co }}$ dominates in Co region for $(6 \AA$ Dy $) /(6 \AA$ $\mathrm{Co})$, and this is understandable from their higher atomic concentrations in their respective regions. But for $(6 \AA$ Dy) $/(3.5 \AA \mathrm{Co}), \sigma_{\text {Dy }}$ dominates through the whole sample and shows maximum value at the central Co region. This feature follows because the Dy moment is disordered at room temperature and the exchange interaction between Co and Dy moments, which is proportional to the Co atomic concentration, makes the Dy moment ordered if the Co atomic concentration is high enough $\left(C_{\mathrm{Co}}>53\right.$ at. \%). In Fig. 7(a) $\sigma_{\text {Dy }}$ shows maximum value at the central region of Co layer because of the higher $\mathrm{Co}$ atomic concentration there.
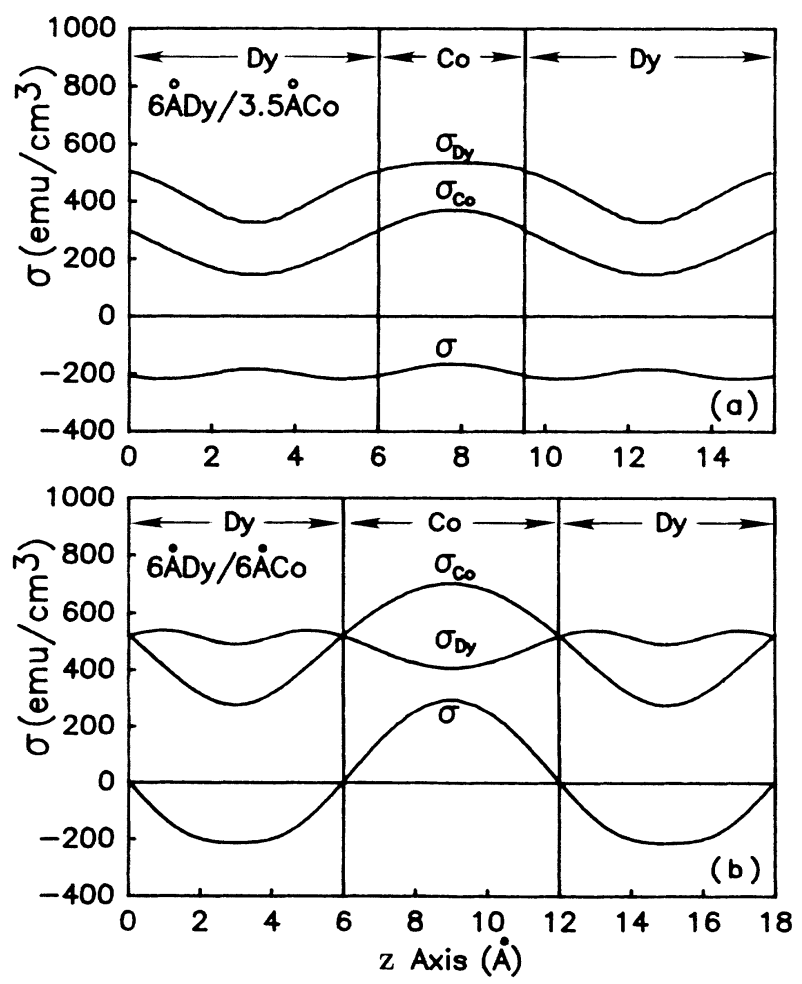

FIG. 7. The magnetization distribution along the film normal for $(6 \AA \mathrm{Dy}) /(3.5 \AA \mathrm{Co})(\mathrm{a})$, and $(6 \AA \mathrm{Dy}) /(6 \AA \mathrm{Co})(\mathrm{b})$. 

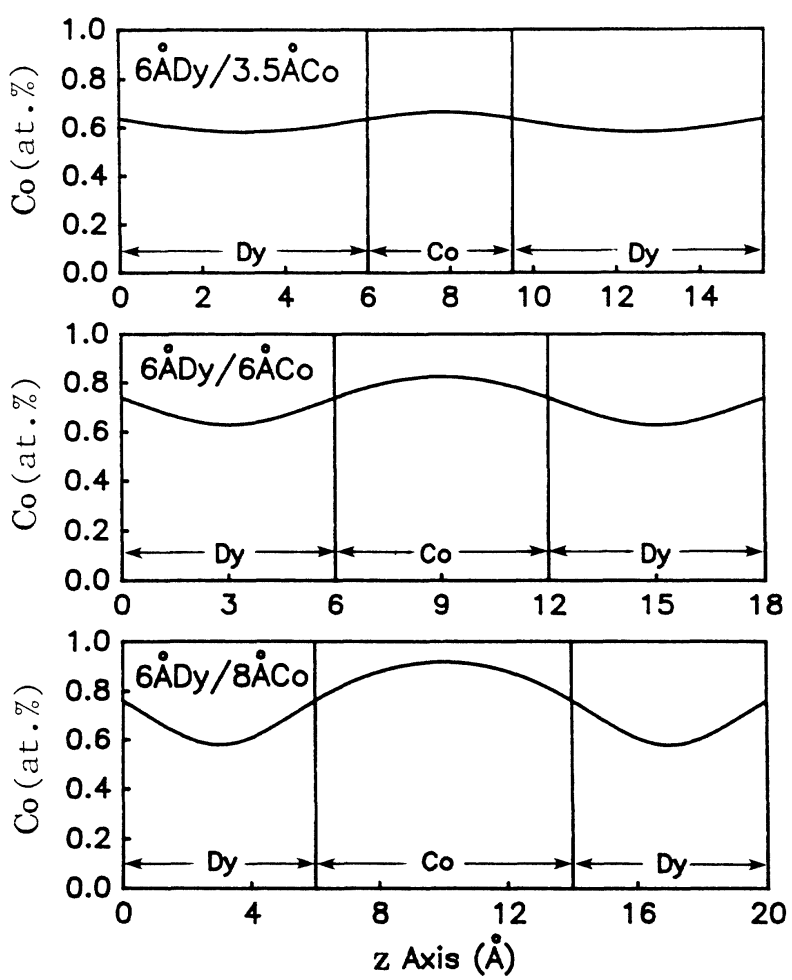

FIG. 8. The Co concentration distribution along the film normal for $(6 \AA \mathrm{Dy}) /(3.5 \AA \mathrm{Co})(\mathrm{a}),(6 \AA \mathrm{Dy}) /(6 \AA \mathrm{Co})(\mathrm{b})$, and $(6 \AA) /(8 \AA \mathrm{Co})(\mathrm{c})$.

The distributions of Co atomic fraction for $(6 \AA$ $\mathrm{Dy}) /(X \AA \mathrm{Co})(X=3.5,6,8)$ are given in Fig. 8. Apparently the $C_{\mathrm{Co}}$ has its maximum value at the center of the Co region and the modulation amplitude increases as the Co layer thickness becomes thicker, and consequently the magnetization changes its value along the film normal as shown in Fig. 7.

Figure 9 shows the Co layer-thickness dependence of the total magnetization $\sigma$ [Fig. 9(a)] and the Co-atomicfraction modulation " $A$ " [Fig. 9(b)]. The average magnetization of Co and Dy subnetworks are also drawn in this figure. It is noticed that the calculated and experimental magnetization data agree very well; the $A$ value is only about 0.1 for the thinnest Co layer thickness of $3.5 \AA$ and its value increases as the Co layer becomes thicker.

The intrinsic anisotropy $K_{u}$, which is expressed with Eq. (11) is fitted to the $K_{u}$ data for $(6 \AA \mathrm{Dy}) /(X \AA \mathrm{Co})$ CMF with $\xi$ as an adjustable parameter and the results are shown in Fig. 10. The agreement between the experimental data and calculated results is remarkable. The fitted value of $\xi=5.26 \times 10^{-6} \mathrm{~cm}$ leads to an average value of the single-ion anisotropy parameter $D=2$ $\times 10^{-17}$ erg, which is reasonable in terms of the typical value of the single-ion random-anisotropy parameter in amorphous alloys. The good agreement between the calculated and experimental data further confirms the suggestion that the Dy-ion anisotropy is the major source of perpendicular anisotropy is reasonable. This also can be found intuitively from the fact that $\left\langle\sigma_{D y}\right\rangle$ curve in Fig. 9(a) is similar to the $K_{u}$ curve in Fig. 8 and $\left\langle\sigma_{\mathrm{Co}}\right\rangle$ curve
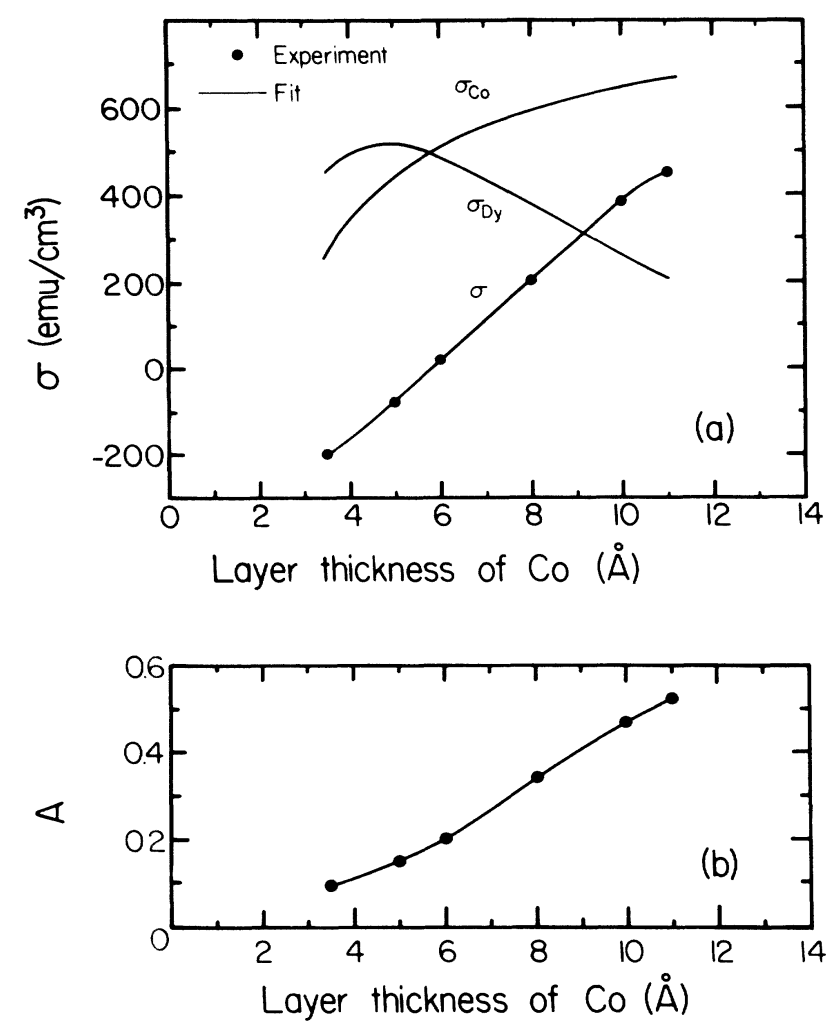

FIG. 9. The comparison of the calculated magnetization with the experimental data for $(6 \AA \mathrm{Dy}) /(X \AA \mathrm{Co})(X=3.5,5,6,8$, 10, and 11) (a), and the Co layer-thickness dependence of the Co atomic fraction modulation (b).

has a totally different shape which hints the Co subnetwork magnetization cannot be the origin of PMA.

\section{B. Analysis for $\mathrm{Tb} / \mathrm{Fe} \mathrm{CMF}^{30}$}

Figure 11 shows the compositional dependence of the total magnetization $\sigma, \mathrm{Tb}$ subnetwork magnetization $\sigma_{\mathrm{Tb}}$, and $\mathrm{Fe}$ subnetwork magnetization $\sigma_{\mathrm{Fe}}$. The $\sigma$ values are basically consistent with those in Ref. 46. $\sigma$ value de-

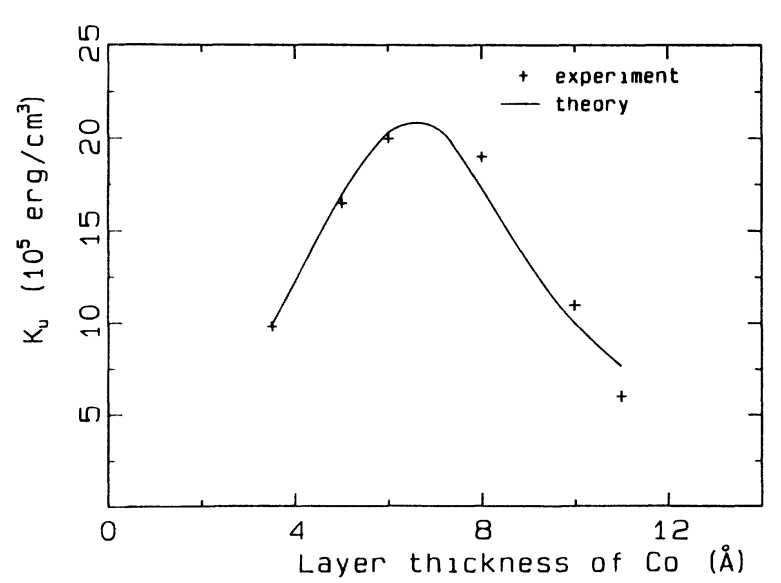

FIG. 10. A comparison between the calculated and exper1mental anisotropy for (6 ̊ Dy)/( $X \AA$ Co $)(X=3.5,6,8,10,11)$. 


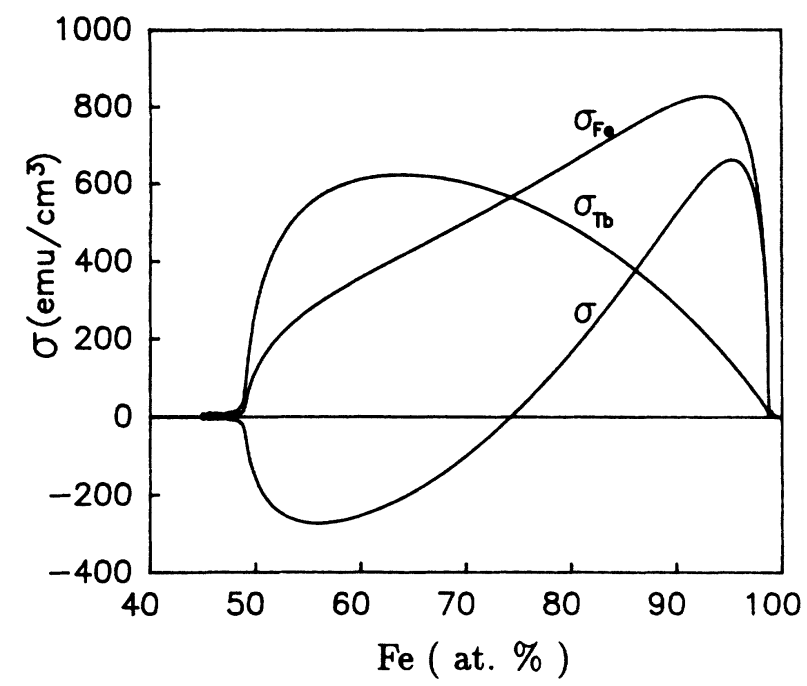

FIG. 11. The Fe concentration dependence of magnetization for $\mathrm{Tb}-\mathrm{Fe}$ alloys: the total magnetization $\sigma_{s}$, Tb-subnetwork magnetization $\sigma_{\mathrm{Tb}}$, and Fe-subnetwork magnetization $\sigma_{\mathrm{Fe}}$.

creases rapidly as $C$ approaches unity because the pure amorphous $\mathrm{Fe}$ is disordered magnetically. ${ }^{47}$ The $\sigma_{\mathrm{Tb}}$ dominates for 53 at. $\%<C<74$ at. $\%$ and $\sigma_{\mathrm{Fe}}$ dominates for $C>74$ at. \%. We notice that both the $\mathrm{Tb}$ - and $\mathrm{Fe}$ subnetwork moments change their value smoothly through the compensation point, i.e., both $\sigma_{\mathrm{Tb}}$ and $\sigma_{\mathrm{Fe}}$ are ordered, but the antiferromagnetic coupling causes the net magnetization to vanish.

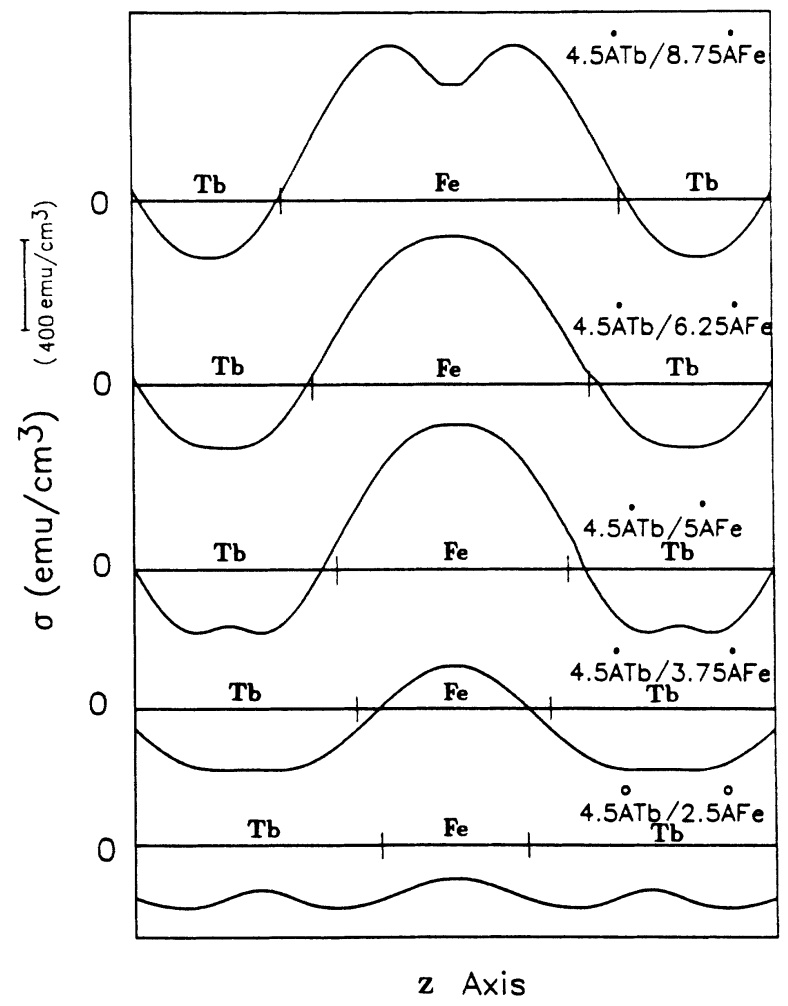

FIG. 12. The magnetization distribution along the film normal for $(4.5 \AA \mathrm{Tb}) /(X \AA \mathrm{Fe})(X=2.5,3.75,5,6.25,8.75)$.

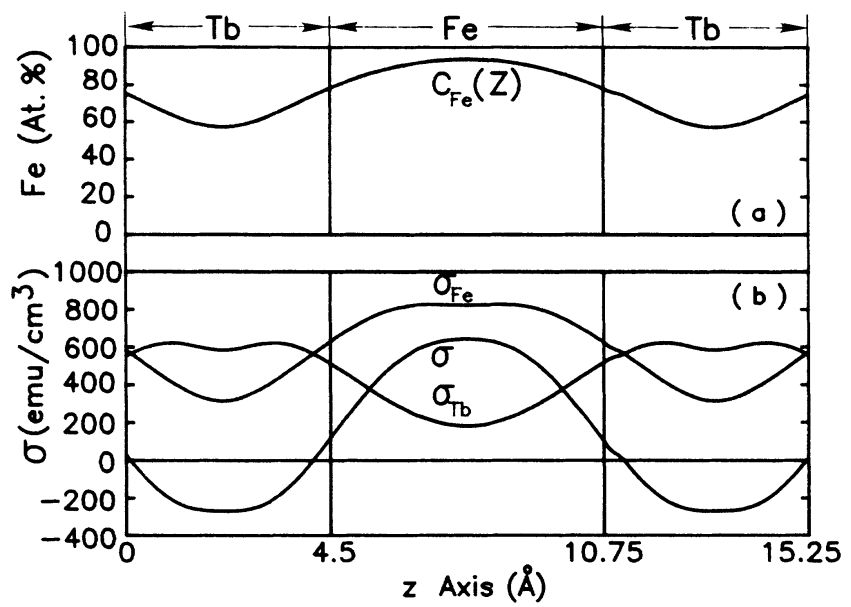

FIG. 13. The distributions of Fe atomic fraction (a), and magnetizations (the total, Tb-subnetwork and Fe-subnetwork magnetizations) for (4.5 ̊ TB)/(6.25 $\AA$ Fe) sample.

The distribution of the magnetization $\sigma$ is illustrated in Fig. 12 for $(4.5 \AA \mathrm{Tb}) /(X \AA \mathrm{Fe})(X=2.5,3.75,5,6.25$, 8.75). It is seen that the Tb subnetwork moment dominates through the whole sample for the sample with the thinnest Fe layer thickness $(X=2.5 \AA)$ and then $\sigma$ has the negative value. As Fe layer thickness increases, the moments of $\mathrm{Tb}$ and $\mathrm{Fe}$ subnetwork dominate in their own region, respectively. It should be pointed out for $(4.5 \AA$ $\mathrm{Tb}) /(8.75 \AA \mathrm{Fe})$ that there is a depression of the $\sigma$ curve at the central $\mathrm{Fe}$ region because the $\mathrm{Fe}$ atomic fraction approaches $98 \%$ there and Fig. 11 has shown the rapid fall of the $\sigma$ curve at this Fe concentration.

An example of the distributions of $\mathrm{Fe}$ atoms and magnetizations $\left(\sigma, \sigma_{\mathrm{Tb}}\right.$, and $\left.\sigma_{\mathrm{Fe}}\right)$ along the film normal for $(4.5 \AA \mathrm{Tb}) /(6.25 \AA \mathrm{Fe})$ is shown in Fig. 13. The $\mathrm{Fe}$ atom distribution $C_{\mathrm{Fe}}(Z)$ has its maximum value of 0.94 at the center of the $\mathrm{Fe}$ region and minimum value of 0.57 at the center of the $\mathrm{Tb}$ region. At the nominal boundary $\sigma$ is equal to zero approximately because $\sigma_{\mathrm{Tb}}$ and $\sigma_{\mathrm{Fe}}$ have about the same magnitude, but they are antiferromagnetically coupled.

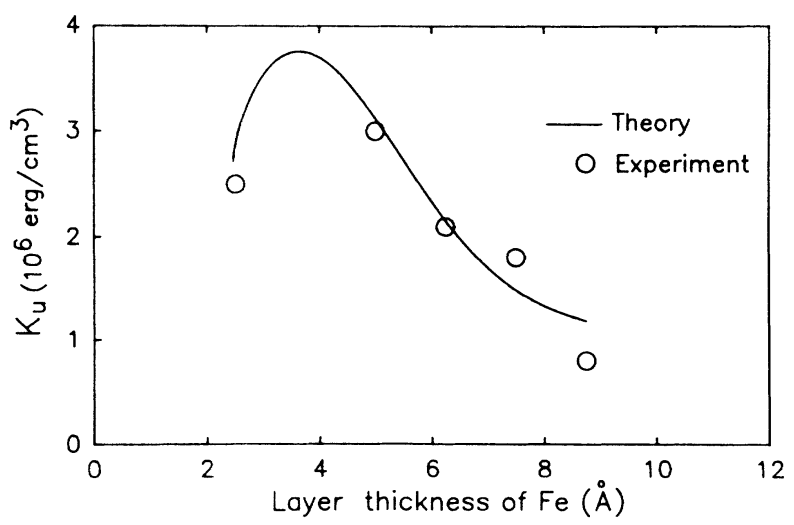

FIG. 14. A comparison between the calculated and experimental anisotropy for $(4.5 \AA \mathrm{Tb}) /(X \AA \mathrm{Ae})(X=2.5,3.3,3.75$, $5,6.25,7.5,8.75$ ) 
Figure 14 shows the Fe layer-thickness dependence of intrinsic anisotropy. The calculated data agree with the experiment fairly well. Since the calculation is based on Eq. (11), this result implies that the model developed in this paper is reasonable.

\section{SUMMARY AND CONCLUSION}

In summary, a detailed model has been developed to understand the occurrence and control of perpendicular anisotropy in $A^{\mathrm{RE}} / \boldsymbol{B}^{\mathrm{TM}} \mathrm{CMF}$. Single-ion anisotropy of the RE ion, which possesses orbital angular momentum, is the major source of PMA and the nominal interface region gives the main contribution to the anisotropy. The distribution of the constituent atoms, the total and constituent magnetization are determined in this model as well. The experimental data only show the magnetic properties resulting from a statistical average over the whole sample; however, the modeling analysis permits the determination of more information about the micromagnetic structure. In this paper we have not investigated in detail the effects of inverse magnetostriction. This remains an important topic for future study. The understanding and control of the microstructure, and thus the magnetic properties may find important applications in various magneto-optic or other data-storage technologies.

\section{ACKNOWLEDGMENTS}

The authors express their thanks to R. D. Kirby and K. D. Aylesworth for assistance and helpful discussion. The research is supported by National Science Foundation (NSF) under Grants No. DMR-86-05367 and No. INT-87-15441.

\section{APPENDIX A: CONSTRAINTS FOR THE DISTRIBUTION FUNCTIONS OF TM AND RE SUBNETWORKS}

The trial distribution should be a sinusoidal curve for the thin-layer CMF. We may imagine that the original distribution of TM and RE are square curves as shown in Figs. 15(b) and 15(c). It is the combination of interdiffusion and the surface roughness that makes the distribution curve sinusoidal.

\section{A. The character of TM distribution function}

(1) The distribution function of normalized TM concentration may be expressed as

$$
\begin{aligned}
\eta_{1}(Z) & =\frac{N_{1}(Z)}{N_{10}} \\
& =\left\{\begin{array}{l}
A_{10}+K A_{1} \sin \left(\frac{\pi}{l_{2}} Z\right) \text { as }-l_{2} \leq Z \leq 0, \\
A_{10}+A_{1} \sin \left(\frac{\pi}{l_{1}} Z\right) \text { as } 0 \leq Z \leq l_{1},
\end{array}\right.
\end{aligned}
$$

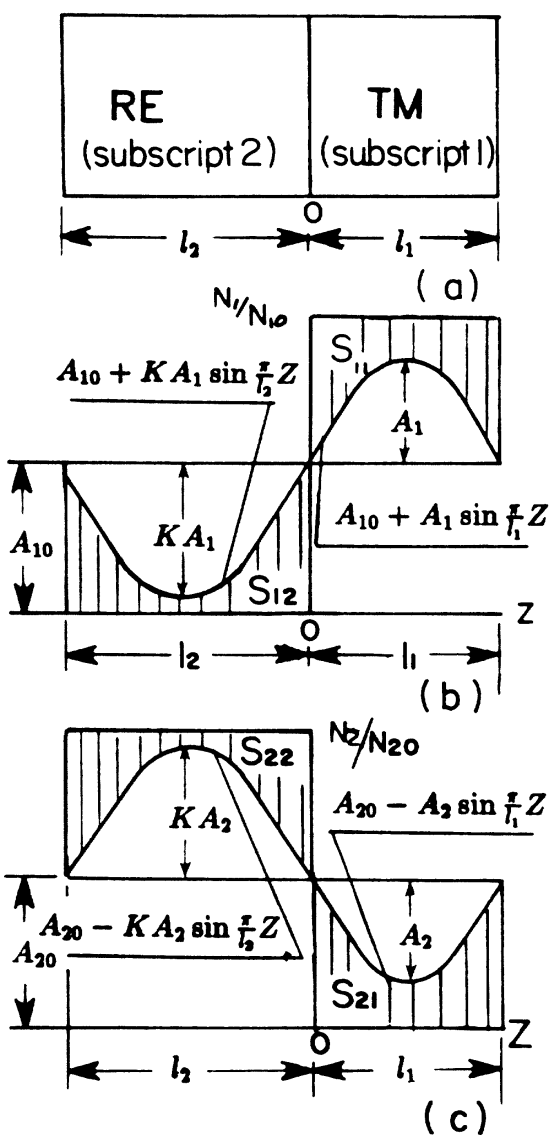

FIG. 15. The constraints between the concentration distributions of TM and RE: the block diagram of $A^{\mathrm{RE}} / B^{\mathrm{TM}}$ bilayer (a), the TM concentration distribution with constraints of $S_{11}=S_{12}$ and $K=l_{2} / l_{1}$ (b), and the Dy concentration distribution with constraints of $S_{21}=S_{22}$ and $K=l_{2} / l_{1}$ (c).

where $N_{10}$ is the concentration of TM (at. number $/ \mathrm{cm}^{3}$ ) and the meaning of parameters $A_{10}, A_{1}, K, l_{1}$, and $l_{2}$ can be found intuitively from Fig. 15 .

(2) Since the TM concentration should vary smoothly from the nominal TM region to the RE region, the first derivative of $\left[N(Z) / N_{10}\right]$ has to be continuous and this leads to

$$
K=\frac{l_{2}}{l_{1}} .
$$

(3) Because of the conservation of TM atom number, there is a constraint between the areas of $S_{11}$ and $S_{12}$, as shown in Fig. 15(b). $S_{11}$ and $S_{12}$ are proportional to the reduced number of TM atom in nominal TM region and the increased number of TM atom in nominal $R E$ region due to the interdiffusion. The constraint requires

$$
S_{11}=S_{12} \text {, }
$$

which leads to

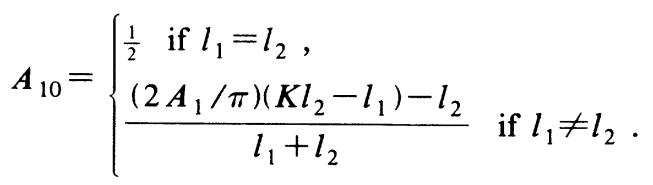




\section{B. The character of the RE distribution function}

Analogous to the TM subnetwork the following expressions exist for RE subnetwork:

$$
\begin{aligned}
\eta_{2} & =\frac{N_{2}(Z)}{N_{20}} \\
& =\left\{\begin{array}{l}
A_{20}-K A_{2} \sin \left(\frac{\pi}{l_{2}} Z\right) \text { if }-l_{2} \leq Z \leq 0, \\
A_{20}-A_{2} \sin \left(\frac{\pi}{l_{1}} Z\right) \text { if } 0 \leq Z \leq l_{1},
\end{array}\right. \\
S_{22} & =S_{21}, \quad \text { if } l_{1} \neq l_{2} . \\
A_{20} & =\left\{\begin{array}{l}
\frac{1}{2} \text { if } l_{1}=l_{2}, \\
\frac{l_{2}-(2 / \pi) A_{2}\left(K l_{2}-l_{1}\right)}{l_{1}+l_{2}}
\end{array}\right.
\end{aligned}
$$

\section{The constraints between TM and RE subnetworks}

If the parameters of $V_{1}$ and $V_{2}$ are defined as the volume per TM atom and the volume per RE atom, respectively, then the following constraint should hold at any position $Z$ :

$$
N_{1}(Z) V_{1}+N_{2} V_{2}=1
$$

since $N_{1} V_{1}$ and $N_{2} V_{2}$ are the volumes of TM and RE atoms in unit volume, respectively.

To simplify Eq. (A8), Eqs. (A1) and (A5) are substituted into Eq. (A8) at $Z=0$, so that Eq. (A8) becomes

$$
A_{10} N_{10} V_{1}+A_{20} N_{20} V_{2}=1 \text {. }
$$

Since $N_{10} V_{1}=N_{20} V_{2}=1$ by their definition, the above equation becomes

$$
A_{10}+A_{20}=1 \text {. }
$$

If this result is substituted into Eqs. (A4) and (A7) we will find

$$
A_{1}=A_{2} \text {. }
$$

Equations (A9) and (A10) are the constraints between TM and RE subnetworks.

Once the parameters $A_{10}, A_{20}, A_{1}$, and $A_{2}$ have been determined, the atomic fraction of TM atoms may be expressed as

$$
C(Z)=\frac{N_{1}(Z)}{N_{1}(Z)+N_{2}(Z)},
$$

and consequently the magnetization values of $\sigma_{s}(Z)$, $\sigma_{1}(Z), \sigma_{2}(Z)$ at $Z$ position can be obtained from Fig. 2 (if $A^{\mathrm{RE}}=\mathrm{Dy}, \quad B^{\mathrm{TM}}=\mathrm{Co}$ ) and Fig. 11 (if $A^{\mathrm{RE}}=\mathrm{Tb}$, $B^{\mathrm{TM}}=\mathrm{Fe}$ ), i.e., the distribution of magnetizations is determined. The rationality of this calculation is confirmed further by the anisotropy calculation where the data of $\sigma_{2}(Z)$ were adopted and the calculated result agreed with experiments fairly well.

\section{APPENDIX B: SINGLE-ION ANISOTROPY IN $A^{\text {RE }} / B^{\text {TM }}$ CMF}

Single-ion anisotropy arises from the interaction between the $4 f$ electron of the RE atom and the local electric field created by the neighboring ions. If the charge distribution of the $4 f$ electrons is nonspherical, i.e., $L \neq 0$, the electric field forces the distribution, the orbital angular momentum, and consequently the magnetic moment into a preferred orientation through the strong spin-orbit coupling. The expressions for the electric potential energy and anisotropy energy of a RE atom are derived on this basis, and the expression for the anisotropy in the CMF is obtained as well.

\section{A. The expression of electric potential energy}

Coulomb interaction $W_{\text {ef }}$ between the $4 f$ electrons and the local electric field can be expressed as ${ }^{25,48}$

$$
\begin{aligned}
& W_{e f}=-|e| \sum_{i} V\left(\mathbf{r}_{i}\right)=-|e| \sum_{i} \sum_{j} \frac{q_{j}}{\left|\mathbf{r}_{j}-\mathbf{r}_{l}\right|} \\
& =-|e| \sum_{l}\left(\sum_{j} q_{j} \sum_{n=1}^{\infty} \frac{r_{l}^{n}}{r_{j}^{n+1}} \frac{4 \pi}{2 n+1} \sum_{m=-n}^{n}(-1)^{m} Y_{n}^{-m}\left(\hat{\mathbf{r}}_{j}\right) Y_{n}^{m}\left(\hat{\mathbf{r}}_{i}\right)\right) \text {, }
\end{aligned}
$$

where $V\left(\mathbf{r}_{i}\right)$ is the electric potential of $4 f$ electron at $\mathbf{r}_{i}$ surrounded by the neighboring ions with charge $q_{j}$ at $\mathbf{r}_{j}$. The summation " $i$ " is over all $4 f$ electrons of a RE ion at the origin [Fig. 6(a)], and summation " $j$ " is over all neighboring ions; usually only the nearest neighbor is taken into account.

The term related to the uniaxial anisotropy along the polar axis $Z_{M}$, which is parallel to the magnetic moment of the RE ion, is that with $n=2, m=0$. Taking this term only, $W_{e f}$ becomes

$$
\begin{aligned}
W_{e f} & =-|e| \sum_{i}\left[\sum_{j} q_{J} \frac{r_{i}^{2}}{r_{j}^{3}} \frac{4 \pi}{5} Y_{2}^{0}\left(\hat{\mathbf{r}}_{j}\right) Y_{2}^{0}\left(\widehat{\mathbf{r}}_{i}\right)\right] \\
& =-\frac{|e|}{4} \sum_{j} \frac{q_{j}\left(3 \cos ^{2} \theta_{j}-1\right)}{r_{j}^{3}} \sum_{i}\left(3 r_{i}^{2} \cos ^{2} \theta_{i}-r_{i}^{2}\right) \\
& =-\frac{|e|}{4} \sum_{j} \frac{q_{j}\left(3 \cos ^{2} \theta_{j}-1\right)}{r_{j}^{3}} \sum_{i}\left(3 Z_{i}^{2}-r_{i}^{2}\right) \\
& =-\frac{|e|}{4} \alpha_{J}\left\langle r^{2}\right\rangle O_{2}^{0} \sum_{j} \frac{q_{j}\left(3 \cos ^{2} \theta_{j}-1\right)}{r_{j}^{3}},
\end{aligned}
$$


where $\theta_{j}$ and $\theta_{i}$ are the angles between $\mathbf{r}_{j}$ and $Z_{M}$ axis, and $\mathbf{r}_{i}$ and $Z_{M}$ axis, respectively.

In the last step of Eq. (B2) the following relationship has been used to simplify the summation over $i$ :

$$
\sum_{i}\left(3 Z_{i}^{2}-r_{i}^{2}\right)=\alpha_{J}\left\langle r^{2}\right\rangle O_{2}^{0}
$$

where $\alpha_{J}$ is Steven's factor and $\left\langle r^{2}\right\rangle$ is the quantummechanical average of the square of the radius of the $4 f$ orbit. $O_{2}^{0}$ is Steven's operator $O_{n}^{m}$ with $n=2$ and $m=0$. It is noticed (i) the values of $\alpha_{J},\left\langle r^{2}\right\rangle$, and $O_{2}^{0}$ are determined by the charge distribution (or wave function) of the $4 f$ electrons. For the rare-earth atom of nonzero orbital angular momentum the spin-orbit coupling $H^{\text {so }}$ $\left(\sim 10^{4} \mathrm{~K}\right)$ is much larger than that of the electric field $H^{e f}\left(\sim 10^{2} \mathrm{~K}\right)$, so that the charge distribution of $4 f$ electron is almost unchanged when the polar axis (or the direction of the magnetic moment of RE ion) changes its direction. This feature will be used to derive the anisotropy expression in the next section. (ii) The summation over $j$ has the meaning of potential at the origin created by all the neighbors. In case of an amorphous structure, the summation over $j$ has to be replaced by an integral weighted with a probability function $P_{j}\left(\mathbf{r}_{j}\right)$, which is the probability to find a $j$ th neighbor ion with charge density $\rho_{j}$ separated from the origin by vector $\mathbf{r}_{j}$ [Fig. 6(a)]. Therefore the electric potential energy $W_{e f}$ may be rewritten as

$$
W_{e f}=-\frac{|e|}{4} \alpha_{J}\left\langle r^{2}\right\rangle O_{2}^{0} \int \rho_{j}\left(3 \cos ^{2} \theta-1\right) P_{j}\left(\mathbf{r}_{j}\right) / r_{j}^{3} d \Omega_{j},
$$

for the amorphous structure. The probability function $P_{j}\left(\mathbf{r}_{j}\right)$ depends strongly on the spatial distribution of RE and TM atoms. In the next section the expressions for $P_{j}\left(\mathbf{r}_{j}\right)$ and subsequently $W_{e f}$ will be derived.

\section{B. The anisotropy for a RE ion in CMF}

\section{The probability function for CMF}

Analogous to the probability function for nominally homogeneous $A^{\mathrm{RE}}-B^{\mathrm{TM}}$ alloys, ${ }^{25,39,40}$ the probability function for CMF may be expressed as

$$
\begin{aligned}
P_{j}\left(\mathbf{r}_{j}\right) & =\eta_{j}\left(Z_{i}+\Delta Z_{i}\right) R\left(r_{j}\right)\left(1+\beta_{j} \cos \alpha_{j}+\cdots\right) \\
& =\left[\eta_{j}\left(Z_{i}\right)+\eta_{j}^{\prime}\left(Z_{i}\right) \Delta Z_{i}\right] R\left(r_{j}\right)\left(1+\beta_{j} \cos \alpha_{j}\right) \\
& =\left[\eta_{j}\left(Z_{i}\right)+\eta_{j}^{\prime}\left(Z_{l}\right) r_{j} \cos \alpha_{j}\right] R\left(r_{j}\right)\left(1+\beta_{j} \cos \alpha_{j}\right),
\end{aligned}
$$

here $\eta_{j}\left(Z_{i}\right)$ is the atomic fraction of RE and TM ions ( $j=1$ refers to TM ion and $j=2$ refers to $\mathrm{RE}$ ion) at $i$ th slice, $R\left(r_{j}\right)$ is the isotropic and $\beta_{j}$ the lowest order of the anisotropic part of the probability function. $\alpha$, is the angle between $\mathbf{r}_{j}$ and the film normal $Z$ (or the easy-axis $Z$ if the CMF has perpendicular anisotropy).

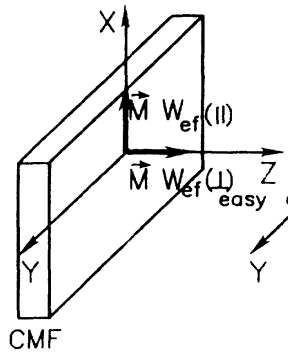

(a)

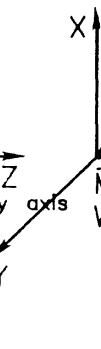

(b)

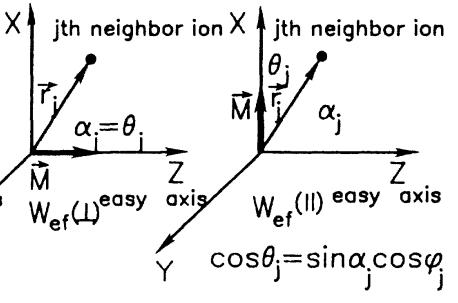

(c)
FIG. 16. The direction of the moment $\mathbf{M}_{1}$ (or $\mathbf{M}_{\|}$) and its corresponding potential energy $W_{e f(1)}\left(W_{e f(\|)}\right)(\mathrm{a})$, the relations between the angles of $\theta_{j}$ and $\alpha_{j}$ as $\mathbf{M}_{1}$ parallel to $Z(\mathrm{~b})$, and $\mathbf{M}_{\|}$ perpendicular to $Z$ (c).

\section{The anisotropy of a $R E$ ion in $C M F$}

a. The general concept of the single-ion anisotropy. As was pointed out above, the electric field forces the magnetic moment into a preferred orientation, i.e., the easy magnetization direction, if the charge distribution of $4 f$ electron is nonspherical. Thus $W_{e f}$ has its minimum value in the easy direction and $W_{\text {ef }}$ increases as the magnetic moment turns away from the easy axis. By the definition the anisotropy energy $K\left(Z_{i}\right)$ per RE ion at $Z_{i}$ is equal to

$$
K\left(Z_{i}\right)=W_{e f(\|)}-W_{e f(1)},
$$

where $W_{e f(\|)}$ is the electric field potential energy as the magnetic moment parallel to the film plane (or perpendicular to the easy axis $Z$ ) and $W_{e f(1)}$ as the magnetic moment parallel to the easy axis $Z$ (or perpendicular to the film plane) as shown in Fig. 16(a).

Equation (B5) shows that the term $\beta_{j} \cos \alpha_{j}$ gives the anisotropic distribution. We notice that the $\cos \theta_{j}$ may be
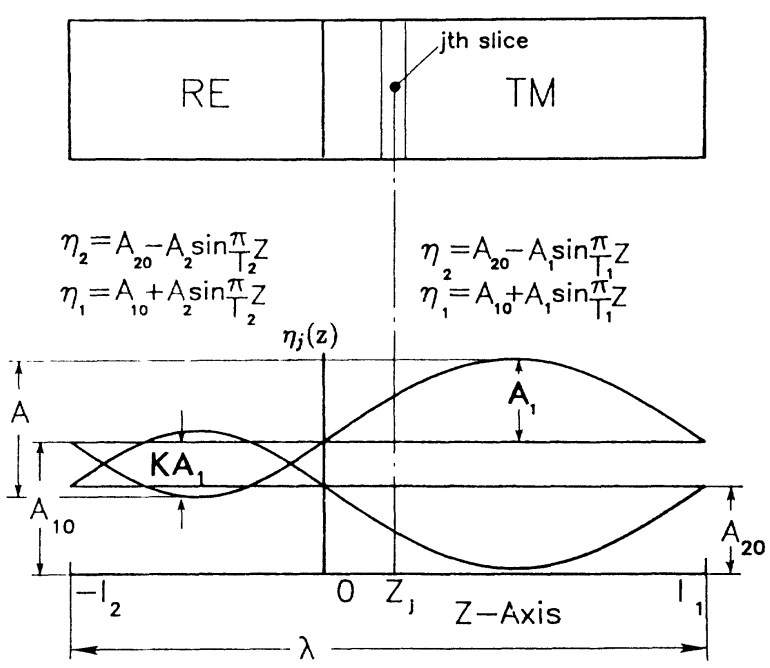

FIG. 17. The concentration distribution $\eta_{j}(Z)\left[\eta_{1}(Z)\right.$ refers to $\mathrm{TM}$ and $\eta_{2}(Z)$ refers to RE]. 
expressed as

$$
\cos \theta_{j}=\cos \alpha_{j}
$$

if the magnetic moment of RE ion along the easy-axis $Z$ [see Fig. 16(b)] and the $W_{e f(1)}$ is going to be calculated. If the magnetic moment of RE ion falls along the film plane [see Fig. 16(c)], then the $\cos \theta_{j}$ may be expressed as

$$
\cos \theta_{j}=\sin \alpha_{j} \cos \phi_{j}
$$

where $\phi_{j}$ is azimuthal angle of the spherical coordinate.

Substituting Eqs. (B7) and (B8) into Eq. (B2) we are able to calculate the $W_{e f(1)}$ and $W_{e f(\|)}$, respectively. b. The calculations of $W_{\text {ef }(\perp)}, W_{\text {ef }(\|)}$, and $K\left(Z_{i}\right)$. The calculation is performed under the following assumptions. (a) The charge density $\rho_{j}$, isotropic parameter $R\left(r_{j}\right)$, anisotropic parameter $\beta_{j}$, and radius $r_{j}(j=1$ refers to TM ion and $j=2$ refers to $\mathrm{RE}$ ion) are constants, but different for TM and RE ions. (b) The RE ion at $Z_{i}$ is surrounded by both of the TM and RE neighbor ions. We assume that the integral in Eq. (B4) is the sum of the integrals performed for $\operatorname{TM}(j=1)$ and $\operatorname{RE}(j=2)$, respectively.

The calculation of $\mathbf{W}_{e f(1)}$ can be performed as follows.

First consider the following integral, which is the main part of Eq. (B4), in spherical coordinates $\left(r_{j}, \alpha_{j}, \varphi_{j}\right)$ :

$$
\begin{aligned}
\mathcal{I}(j) & =\int \rho_{j}\left(3 \cos ^{2} \theta_{j}-1\right) \frac{P_{j}\left(\mathbf{r}_{j}\right)}{r_{j}^{3}} d \Omega_{j} \\
& =\int \rho_{j}\left(3 \cos ^{2} \theta_{j}-1\right)\left[\eta_{j}\left(Z_{i}\right)+\eta_{j}^{\prime}\left(Z_{i}\right) r_{j} \cos \alpha_{j}\right] \frac{R\left(r_{j}\right)\left(1+\beta_{j} \cos \alpha_{j}\right)}{r_{j}^{3}} d \Omega_{J} \\
& =\int \rho_{J}\left(3 \cos ^{2} \alpha_{j}-1\right)\left[\eta_{j}\left(Z_{i}\right)+\eta_{j}^{\prime}\left(Z_{l}\right) r_{i} \cos \alpha_{j}\right] \frac{R\left(r_{j}\right)\left(1+\beta_{j} \cos \alpha_{j}\right)}{r_{j}^{3}} d \Omega_{J} .
\end{aligned}
$$

In the last step of the above derivation Eq. (B7) has been used. After a tedious integration the above equation can be simplified as

$$
\mathcal{I}(j)=\frac{16}{15} \pi\left(\frac{\rho_{j} R\left(r_{j}\right) \beta_{j}}{r_{j}^{3}}\right) \eta_{j}^{\prime}\left(Z_{l}\right)
$$

Using definitions of $W_{e f(1)}$ and Eq. (B4), $W_{e f(1)}$ can be expressed as

$$
\begin{aligned}
W_{e f(1)} & =-\frac{|e|}{4} \alpha_{J}\left\langle r^{2}\right\rangle O_{2}^{0}[\mathcal{I}(1)+\mathcal{I}(2)] \\
& =-\frac{|e|}{4} \alpha_{J}\left\langle r^{2}\right\rangle O_{2}^{0} \frac{16}{15} \pi\left[\left(\frac{\rho_{1} R\left(r_{1}\right) \beta_{1}}{r_{1}^{2}}\right) \eta_{1}^{\prime}\left(Z_{i}\right)+\left(\frac{\rho_{2} R\left(r_{2}\right) \beta_{2}}{r_{2}^{2}}\right) \eta_{2}^{\prime}\left(Z_{l}\right)\right] \\
& =-\frac{|e|}{4} \alpha_{J}\left\langle r^{2}\right\rangle O_{2}^{0} \frac{16}{15} \pi\left[\left(\frac{\rho_{1} R\left(r_{1}\right) \beta_{1}}{r_{1}^{2}}\right)-\left(\frac{\rho_{2} R\left(r_{2}\right) \beta_{2}}{r_{2}^{2}}\right)\right] \eta_{1}^{\prime}\left(Z_{l}\right) .
\end{aligned}
$$

In the last step of the above derivation, the relation $\eta_{2}^{\prime}\left(Z_{i}\right)=-\eta_{1}^{\prime}\left(Z_{i}\right)$ has been used.

The calculation of $W_{e f(\|)}$ can be performed similarly. We consider the following integral in spherical coordinates $\left(r_{j}, \alpha_{j}, \varphi_{J}\right)$ :

$$
\mathcal{J}(j)=\int \rho_{j}\left(3 \cos ^{2} \theta_{j}-1\right) \frac{P_{j}\left(\mathbf{r}_{j}\right)}{r_{j}^{3}} d \Omega_{j}=\int \rho_{j}\left(3 \sin ^{2} \alpha_{j} \cos ^{2} \varphi_{j}-1\right)\left[\eta_{j}\left(Z_{l}\right)+\eta_{j}^{\prime}\left(Z_{i}\right) r_{j} \cos \alpha_{j}\right] \frac{R\left(r_{j}\right)\left(1+\beta_{j} \cos \alpha_{j}\right)}{r_{j}^{3}} d \Omega_{j} .
$$

In the last step of the above derivation Eq. (B8) has been used. After the tedious integration the above equation can be simplified to

$$
\mathcal{J}(j)=-\frac{8}{15} \pi\left(\frac{\rho_{j} R\left(r_{j}\right) \beta_{j}}{r_{j}^{2}}\right) \eta_{j}^{\prime}\left(Z_{i}\right)
$$

Using the definition of $W_{e f(\|)}$ and Eq. (B4), $W_{e f(\|)}$ may be expressed as

$$
W_{e f(\|)}=-\frac{|e|}{4} \alpha_{J}\left\langle r^{2}\right\rangle O_{2}^{0}[\mathcal{J}(1)+\mathcal{J}(2)]=+\frac{|e|}{4} \alpha_{J}\left\langle r^{2}\right\rangle O_{2}^{0} \frac{8}{15} \pi\left(\frac{\rho_{1} R\left(r_{1}\right) \beta_{1}}{r_{1}^{2}}-\frac{\rho_{1} R\left(r_{2}\right) R\left(r_{2}\right) \beta_{2}}{r_{2}^{2}}\right) \eta^{\prime}\left(Z_{i}\right) .
$$

In the last step of the above derivation, the relation $\eta_{2}^{\prime}\left(Z_{i}\right)=-\eta_{1}^{\prime}\left(Z_{1}\right)$ has been used as well.

Comparing Eq. (B14) with Eq. (B11) we find 


$$
W_{e f(\|)}=-\frac{W_{e f(1)}}{2} .
$$

The anisotropy energy $K\left(Z_{l}\right)$ per RE ion at $Z_{l}$ is equal to

$$
K\left(Z_{l}\right)=W_{e f(\|)}-W_{e f(1)}=-\frac{3}{2} W_{e f(1)} .
$$

Substituting Eq. (B11) into Eq. (B16), the anisotropy of a $R E$ ion in $C M F K\left(Z_{l}\right.$ ) can be expressed as

$$
K\left(Z_{i}\right)=\frac{2 \pi|e|}{5} \alpha_{J}\left\langle r^{2}\right\rangle O_{2}^{0}\left(\frac{\rho_{1} R\left(r_{1}\right) \beta_{1}}{r_{1}^{2}}-\frac{\rho_{2} R\left(r_{2}\right) \beta_{2}}{r_{2}^{2}}\right) \eta_{1}^{\prime}\left(Z_{l}\right) .
$$

In Eq. (B17) $O_{2}^{0}$ can be expressed as $\left(2 J_{Z}^{2}\right)$ if $Z$ is the easy axis. Thus the local anisotropy energy $K\left(Z_{l}\right)$ per $\mathrm{RE}$ ion is

$$
K\left(Z_{i}\right)=K_{0}\left(\sigma_{Z_{l}}^{2}\right) \eta_{1}^{\prime}\left(Z_{\imath}\right)
$$

where

$$
K_{0}=\frac{4 \pi|e|}{5 N^{2} \mu_{B}^{2} g_{2}^{2}}\left(\frac{\rho_{1} R\left(r_{1}\right) \beta_{1}}{r_{1}^{2}}-\frac{\rho_{2} R\left(r_{2}\right) \beta_{2}}{r_{2}^{2}}\right) \alpha_{J}\left\langle r^{2}\right\rangle
$$

is treated as a constant for given $A^{\mathrm{RE}}-B^{\mathrm{TM}}$ alloy, and the relation $J_{Z}=\sigma_{Z} / N \mu_{B} g_{2}$ has been used.

\section{The anisotropy for $A^{\mathrm{RE}} / B^{\mathrm{TM}} \mathrm{CMF}$}

The anisotropy of the $A^{\mathrm{RE}} / B^{\mathrm{TM}} \mathrm{CMF}$ is the average of $K\left(Z_{l}\right)$ throughout the whole volume, i.e., see Fig. 17:

$$
K_{u}=\left\langle K\left(Z_{l}\right)\right\rangle=\frac{2}{\lambda} \int_{-l_{2} / 2}^{l_{1} / 2} N_{20} \eta_{2}\left(Z_{l}\right) K\left(Z_{i}\right) d Z_{l}=\frac{2}{\lambda} \int_{-l_{2} / 2}^{l_{1} / 2} N_{20} \eta_{2}(Z) K_{0}\left(\sigma_{Z}^{2}\right) \eta_{1}^{\prime}(Z) d Z \text {. }
$$

In the last step of the above derivation, the subscript " $i$ " is omitted. The expressions of $\eta_{2}(Z)$ and $\eta_{1}(Z)$ [or consequently $\eta_{1}^{\prime}(Z)$ ] can be found in Fig. 17.

Because the parameters $A_{1}, A_{10}, A_{20}$, and $\sigma_{Z}$ can be obtained in terms of mean-field model as shown in the text and Appendix A, the anisotropy $K_{u}$ may be calculated numerically if the constant $K_{0}$ is regarded as an adjustable parameter.

If the magnetization $\sigma_{Z}$ changes smoothly, which is true for the sample with thin layer thicknesses $l_{1}$ and $l_{2}$, the $K_{u}$ expression may be rewritten as

$$
\begin{aligned}
& K_{u}= \frac{2}{\lambda} N_{20} K_{0}\left\langle\sigma_{Z}^{2}\right\rangle \int_{-l_{2} / 2}^{l_{1} / 2} \eta_{2}(Z) \eta_{1}^{\prime}(Z) d Z \\
&=\frac{2}{\lambda} N_{20} K_{0}\left\langle\sigma_{Z}^{2}\right\rangle\left\{\int_{-l_{2} / 2}^{0}\left[A_{20}-K A_{1} \sin \left(\frac{\pi}{l_{2}} Z\right]\right] \frac{\pi}{l_{2}} K A_{2} \cos \left(\frac{\pi}{l_{2}} Z\right) d Z\right. \\
&\left.+\int_{0}^{l_{1} / 2}\left[A_{20}-A_{1} \sin \left(\frac{\pi}{l_{1}} Z\right)\right] \frac{\pi}{l_{1}} A_{1} \cos \left(\frac{\pi}{l_{1}} Z\right) d Z\right\} .
\end{aligned}
$$

After lengthy operation the anisotropy $K_{u}$ for CMF may be expressed as

$$
K_{u} \simeq 2 N_{20} K_{0}\left\langle\sigma_{Z}^{2}\right\rangle A_{20} \frac{A}{\lambda}\left(1-\frac{A_{1}(1-K)}{2 A_{20}}\right) .
$$

$$
K_{u} \simeq 2 N_{20} K_{0}\left\langle\sigma_{Z}^{2}\right\rangle A_{20} \frac{A}{\lambda}
$$

as $K=1$, i.e., TM and RE have equal layer thicknesses. This is the case of equal RE and TM layer thicknesses which has been pointed out in Eq. (18).
${ }^{*}$ Permanent address: Department of Electronic Engineering, Hangzhou University, Hangzhou, Zhejiang, People's Republic of China.

${ }^{\dagger}$ Present address: Institute of Physics, Chinese Academy of Sciences, P. O. Box 603, Beijing 100080, People's Republic of China.

${ }^{1}$ N. Sato, J. Appl. Phys. 59, 2514 (1986).
${ }^{2}$ N. Sato and K. Habu, J. Appl. Phys. 61, 4287 (1987).

${ }^{3}$ K. Yamauchi, K. Habu, and N. Sato, J. Appl. Phys. 64, 5478 (1988).

${ }^{4}$ N. Sato, K. Habu, and T. Oyama, IEEE Trans. Magn. MAG-23, 2614 (1987).

5S. Honda, M. Nawate, M. Yoshiyama, and T. Kuda, IEEE Trans. Magn. MAG-23, 2952 (1987). 
${ }^{6}$ S. C. Shin, M. L. Cofied, and R. H. D. Nuttall, J. Appl. Phys. 61, 4326 (1987).

${ }^{7}$ Y. Suzuki, H. Masuda, T. Shibata, and N. Koshizuka, IEEE Trans. Magn. MAG-23, 3704 (1987).

${ }^{8}$ D. J. Sellmyer, Z. R. Zhao, Z. S. Shan, and S. Nafis, J. Appl. Phys. 61, 4323 (1987).

${ }^{9}$ Z. S. Shan, S. Nafis, K. D. Ayleworth, and D. J. Sellmyer, J. Appl. Phys. 63, 3218 (1988).

${ }^{10}$ Z. S. Shan and D. J. Sellmyer, J. Appl. Phys. 64, 5745 (1988).

${ }^{11}$ D. J. Sellmyer and Z. S. Shan, Proceeding of the Materials Research Society, International Meeting on Advanced Materials, Vol. 10, Multilayers Symposium, Tokyo, 269 (1989).

${ }^{12}$ D. J. Sellmyer, J. A. Woollam, Z. S. Shan, and W. A. McGahan, Mat. Res. Soc. Symp. Proc., Materials for Magneto-Optic Data Storage, edited by C. J. Robinson et al., 51, (1989).

${ }^{13}$ Z. S. Shan, D. J. Sellmyer, S. S. Jaswal, Y. J. Wang, and J. X. Shen, Phys. Rev. Lett. 63, 449 (1989).

${ }^{14}$ Z. S. Shan, Ph.D. Dissertation, University of Nebraska, 1990.

${ }^{15}$ P. F. Carcia, A. D. Meinhaldt, and A. Suna, Appl. Phys. Lett. 47, 178 (1985).

${ }^{16}$ P. F. Carcia, J. Vac. Sci. Technol. A5, 1975 (1987).

${ }^{17}$ P. F. Carcia, J. Appl. Phys. 63, 5066 (1988).

${ }^{18}$ F. J. A. den Broeder, H. C. Donkersloot, H. J. G. Draaisma, and W. J. M. de Jonge, J. Appl. Phys. 61, 4317 (1987).

${ }^{19}$ H. J. G. Draaisma, W. J. M. de Jonge, F. J. A. den Broder, J. Magn. Magn. Mater. 66, 351 (1987).

${ }^{20}$ F. J. A. den Broeder, D. Kuiper, and H. J. G. Draaisma, IEEE Trans. Magn. MAG-23, 3696 (1987).

${ }^{21}$ H. J. G. Draaisma, F. J. A. den Broeder, and W. J. M. de Jonge, J. Appl. Phys. 63, 3479 (1988).

${ }^{22}$ H. J. G. Draaisma and W. J. M. de Jonge, J. Appl. Phys. 62, 3318 (1987).

${ }^{23}$ F. J. A. den Broeder, D. Kuiper, A. P. Van de Mosselaer, and W. Hoving, Phys. Rev. Lett. 60, 2769 (1988).

${ }^{24}$ N. C. Koon, B. T. Jonker, F. A. Volkening, J. J. Krebs, and G. A. Prinz, Phys. Rev. Lett. 59, 2463 (1987).

${ }^{25}$ Y. Suzuki, S. Takyma, F. Kirino, and N. Ohta, IEEE Trans. Magn. MAG-23, 2275 (1987).

${ }^{26}$ Y. Suzuki and N. Ohta, J. Appl. Phys. 63, 3633 (1988).

${ }^{27}$ A. Yoshihara, M. Takahashi, T. Shimamori, T. Wakiyama, M. Miyazaki, S. Yamaguchi, and K. Hayashi, J. Appl. Phys.
49, 5492 (1978)

${ }^{28}$ T. Miyazaki, K. Hayashi, S. Yamaguchi, M. Takahashi, A. Yoshihara, T. Shimaori, and T. Wakiyama, J. Magn. Magn. Mater. 75, 243 (1988).

${ }^{29}$ M. Takahashi, A. Yoshiara, T. Shimamori, T. Wakiyama, T. Miyazaki, K. Hayashi, and S. Yamaguchi, J. Magn. Magn. Mater. 75, 252 (1988).

${ }^{30}$ Z. S. Shan and D. J. Sellmyer, J. Appl. Phys. 67, 5713 (1990).

${ }^{31}$ R. Hasegawa, J. Appl. Phys. 46, 5263 (1975).

${ }^{32}$ A. Gangulee and R. C. Taylor, J. Appl. Phys. 49, 1762 (1978).

${ }^{33}$ A. Gangulee and R. J. Kobliska, J. Appl. Phys. 49, 4169 (1978).

${ }^{34}$ A. Gangulee and R. J. Kobliska, J. Appl. Phys. 49, 4896 (1978).

${ }^{35}$ M. Mansuripur and M. F. Ruane, IEEE Trans. Magn. MAG-22, 33 (1986).

${ }^{36}$ C. G. Robbins (unpublished).

${ }^{37}$ T. Egami, C. D. Graham, Jr., W. Dmowski, P. Zhou, P. J. Flanders, E. E. Marinero, H. Notrys, and C. Robinson, IEEE Trans. Magn. MAG-23, 2269 (1987).

${ }^{38}$ Y. Suzuki, J. Haimovich, and T. Egami, Phys. Rev. B 35, 2162 (1987).

${ }^{39}$ G. S. Cargill III and T. Mizoguchi, J. Appl. Phys. 49, 1753 (1978).

${ }^{40}$ T. Mizoguchi and G. S. Cargill III, J. Appl. Phys. 50, 3570 (1979).

${ }^{41}$ K. H. J. Bushow, J. Appl. Phys. 63, 3130 (1988).

${ }^{42}$ L. T. Baczewski, M. Piecuch, J. Durand, G. Marchal, and P. Delcroix, Phys. Rev. B 40, 11237 (1989).

${ }^{43}$ A. Fert, in Proceedings of the NATO Advanced Study Institute, Crete, Greece (to be published).

${ }^{44}$ C. H. Lee, Hui He, F. J. Lamelas, W. Vavra, C. Uher, and R. Clarke, Phys. Rev. B 42, 1066 (1990).

${ }^{45}$ F. J. A. den Broeder, Proceedings of the E-MRS Conference (to be published).

${ }^{46}$ P. Hansen, C. Clausen, G. Much, M. Rosenkraz, and K. Witter, J. Appl. Phys. 66, 756 (1989).

${ }^{47}$ J. J. Rhyne, IEEE Trans. Magn. MAG-21, 1990 (1985).

${ }^{48}$ M. T. Hutchings, in Solid State Physics, edited by F. Seitz and D. Turnbull (Academic, New York, 1964), Vol. 16, pp. 227-273 